\title{
Effect of N, C, and B interstitials on the structural and magnetic properties of alloys with $\mathrm{Cu}_{3} \mathrm{Au}$ structure
}

\author{
Ingo Opahle, ${ }^{1, *}$ Harish K. Singh, ${ }^{1}$ Jan Zemen, ${ }^{2,3}$ Oliver Gutfleisch $\odot,{ }^{1}$ and Hongbin Zhang ${ }^{1}$ \\ ${ }^{1}$ Institute of Materials Science, TU Darmstadt, 64287 Darmstadt, Germany \\ ${ }^{2}$ Faculty of Electrical Engineering, Czech Technical University in Prague, Technicka 2, Prague 166 27, Czech Republic \\ ${ }^{3}$ Department of Physics, Blackett Laboratory, Imperial College London, London SW7 2AZ, United Kingdom
}

(Received 3 January 2020; accepted 25 March 2020; published 6 May 2020)

\begin{abstract}
High-throughput density functional calculations are used to investigate the effect of interstitial B, C, and N atoms on 21 alloys reported to crystallize in the cubic $\mathrm{Cu}_{3} \mathrm{Au}$ structure. It is shown that the interstitials can have a significant impact on the magnetocrystalline anisotropy energy (MAE), the thermodynamic stability, and the magnetic ground-state structure, making these alloys interesting for hard magnetic, magnetocaloric, and other applications. For 29 alloy-interstitial combinations the formation of stable alloys with interstitial concentrations above $5 \%$ is expected. $\mathrm{In} \mathrm{Ni}_{3} \mathrm{Mn}$ interstitial $\mathrm{N}$ induces a tetragonal distortion with substantial uniaxial MAE for realistic $\mathrm{N}$ concentrations. $\mathrm{Mn}_{3} X \mathrm{~N}_{x}(X=\mathrm{Rh}, \mathrm{Ir}, \mathrm{Pt}$, and $\mathrm{Sb})$ compounds are identified as alloys with strong magnetocrystalline anisotropy. For $\mathrm{Mn}_{3} \mathrm{Ir}$ we find a strong enhancement of the MAE upon $\mathrm{N}$ alloying in the most stable collinear ferrimagnetic state as well as in the noncollinear magnetic ground state. $\mathrm{Mn}_{3} \operatorname{Ir}_{\mathrm{and}} \mathrm{Mn}_{3} \operatorname{IrN}$ also show interesting topological transport properties. The effects of $\mathrm{N}$ concentration and strain on the magnetic properties are discussed. Further, the huge impact of $\mathrm{N}$ on the MAE of $\mathrm{Mn}_{3} \mathrm{Ir}$ and a possible impact of interstitial $\mathrm{N}$ on amorphous $\mathrm{Mn}_{3} \mathrm{Ir}$, a material that is indispensable in today's data storage devices, are discussed in relation to the electronic structure. For $\mathrm{Mn}_{3} \mathrm{Sb}$, noncollinear, ferrimagnetic, and ferromagnetic states are very close in energy, making this material potentially interesting for magnetocaloric applications. For the investigated $\mathrm{Mn}$ alloys and competing phases, the determination of the magnetic ground state is essential for a reliable prediction of the phase stability.
\end{abstract}

DOI: 10.1103/PhysRevResearch.2.023134

\section{INTRODUCTION}

Magnetic materials with a large magnetocrystalline anisotropy energy (MAE) are an important component in a wide range of everyday devices. Permanent magnets are used, for example, in hard disks, electromotors, and direct-drive wind turbines. Antiferromagnetic compounds find applications in exchange-biased films for data storage devices and magnetic random access memories and are of interest for spintronics applications [1-3]. Present high-performance magnets like $\mathrm{Nd}-\mathrm{Fe}-\mathrm{B}$ contain a considerable amount of rare-earth elements, which in recent years have been subject to strong price fluctuations. The development of alternative permanent magnets without the use of critical rare-earth elements is therefore highly important [4-6]. Basic requirements for a good permanent magnet material are a large uniaxial MAE, a large saturation magnetization, and a high Curie temperature.

High-throughput calculations in the framework of densityfunctional theory (DFT) have become an increasingly

\footnotetext{
*opahle@tmm.tu-darmstadt.de

Published by the American Physical Society under the terms of the Creative Commons Attribution 4.0 International license. Further distribution of this work must maintain attribution to the author(s) and the published article's title, journal citation, and DOI.
}

important tool for the design of new functional materials in recent years $[7,8]$. In addition to increased computational resources, this is due to the development of large databases and new concepts like efficient descriptors. A number of studies have focused on the development of improved materials for batteries [9,10], thermoelectrics [11-13], and photovoltaics [14].

For magnetic materials, despite their technological importance, computational high-throughput studies are scarce. Bocarsly et al. performed a screening of potential magnetocaloric materials [15], and Elsässer and colleagues performed a screening of potential hard magnetic rare-earth lean compounds [16-18]. Other studies focused on potential rare-earth free permanent magnets [19-22] or the prediction of new magnetic compounds [23-26]. A complete study of the stability, magnetic properties, and MAE of potential new magnetic compounds taking into account competing phases and magnetic structures has not, to the best of our knowledge, been performed up to now.

Theoretical high-throughput descriptions of functional magnetic materials involve different energy scales like Coulomb correlations, exchange interactions, and magnetocrystalline anisotropy. Antiferromagnetic (AF) order can have a strong impact on the total energy and phase stability of Mn alloys [24,27], which are of interest for hardmagnetic [28], magnetocaloric [29], and spintronics applications [2]. The effect of AF interactions on the energetics 
has been explored in high-throughput calculations for certain classes of compounds with known magnetic structure prototypes $[24,30,31]$ and on selected compounds using evolutionary algorithms $[25,32]$ or a symmetry-based prioritization [33]. Complex (noncollinear) structures and nonferromagnetic ground states of competing structures in a phase diagram have not been studied up to now, as the computational effort is easily enlarged by one order of magnitude and, often, suitable magnetic structure prototypes are not known. For the calculation of the MAE, which sets an upper limit to the coercivity, a proper treatment of spin-orbit coupling (SOC) and a high numerical accuracy, especially a careful convergence of $k$-space integrals, are essential. Rare-earth compounds with localized $4 f$ electrons require a careful treatment of Coulomb correlations, where accurate methods suitable for a high-throughput screening still have to be developed. While individual aspects have been carefully addressed in the literature, the complexity of interactions makes a highthroughput prediction of functional magnetic materials still highly demanding.

It has been shown in the literature that interstitials can have a strong impact on the magnetic properties, including the magnetocrystalline anisotropy energy [34-36], the magnetic structure [37], and magnetostructural phase transitions $[38,39]$. The $\mathrm{Cu}_{3} \mathrm{Au}$ structure is one of the most common structure types, with more than 200 reported compounds. Ferromagnetic compounds like $\mathrm{Fe}_{3} \mathrm{Pt}$ can exhibit a large magnetization, which makes them interesting for applications in exchange-coupled magnets $[40,41]$. Due to the cubic symmetry they have, however, only a relatively small MAE. In contrast, antiferromagnetic compounds like $\mathrm{Mn}_{3} \mathrm{Ir}$ can exhibit a huge magnetocrystalline anisotropy [42] but have vanishing magnetization. This is used in exchange-biased films, where giant exchange anisotropies are reported [43,44]. Further, Mnrich alloys of $\mathrm{Cu}_{3} \mathrm{Au}$ prototype have attracted attention due to a large magnetostriction [45] and Berry-curvature-related phenomena like a large anomalous Hall effect and a facetdependent spin Hall effect [46-48].

In this paper, we investigate the effect of interstitial $\mathrm{N}$, $\mathrm{C}$, and $\mathrm{B}$ atoms on the stability and magnetic properties of 21 experimentally reported compounds with cubic $\mathrm{Cu}_{3} \mathrm{Au}$ structure and the magnetic $3 d$ elements $\mathrm{Cr}, \mathrm{Mn}, \mathrm{Fe}, \mathrm{Co}$, and Ni. To identify the magnetic ground state of Mn alloys, the energy of different spin structures with maximal magnetic subgroup symmetry is calculated, which according to Ref. [49] accounts for the majority of measured magnetic structures on the Bilbao Crystallographic Server. This allows us to identify also complex noncollinear (NC) ground states, which are frequently observed in Mn alloys. In total, about 1000 magnetic configurations are probed to obtain reliable energies for alloys relevant for the phase stability of binary and ternary $\mathrm{Mn}$ alloy systems related to this study (Fig. 1). Stable interstitial phases are identified taking into account all possible decomposition reactions. We show that interstitials can lead to a strong enhancement of the magnetocrystalline anisotropy energy in these alloys and that high-throughput DFT calculations are able to identify hard magnetic materials even in the presence of complex magnetic interactions. (a)

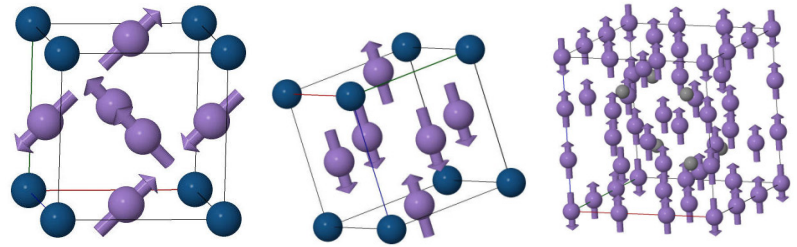

(b)

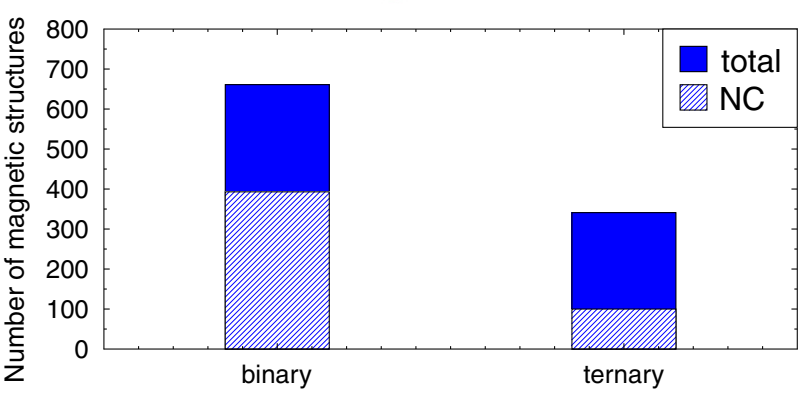

FIG. 1. (a) Examples for automatically generated initial spin structures: a NC structure and collinear ferrimagnetic structure for $\mathrm{Mn}_{3} \mathrm{Ir}$ and a collinear ferrimagnetic structure for $\mathrm{Mn}_{23} \mathrm{C}_{6}$ (from left to right). (b) Total number of (solid blue) and number of $\mathrm{NC}$ (hatched blue) magnetic structures calculated for binary and ternary Mn alloys in this work.

\section{COMPUTATIONAL DETAILS}

Calculations were performed within density-functional theory using an extended version of the high-throughput environment (HTE) [50,51] employing the VASP [52,53] and FPLO [54] codes. For the present investigation, the HTE has been extended for the calculation of magnetic properties such as the investigation of different spin configurations and the calculation of the MAE. Further, an automatic detection of suitable interstitial positions was implemented.

For structure optimizations and the calculation of formation energies the VASP code [52,53] has been used following an approach similar to that in Ref. [51]. For the final results shown here projector augmented-wave pseudopotentials with a cutoff energy $E=500 \mathrm{eV}$ and constant density of $k$ points corresponding, e.g., to a $k$ mesh of $14 \times 14 \times 14 k$ points in the full Brillouin zone (FBZ) for $\mathrm{Mn}_{3} \mathrm{Sb}$ were used. For MAE calculations, the full-potential local-orbital method [54] (FPLO, version 14-49) was used, employing the optimized crystal and (collinear) magnetic structure obtained in the preceding VASP calculations. In the FPLO code, accurate fullpotential calculations are combined with the solution of the four-component Kohn-Sham-Dirac equation, which implicitly contains spin-orbit coupling up to all orders.

As a descriptor for hard magnetic alloys the magnetic force theorem with a moderate $k$ mesh (up to $20 \times 20 \times 20$ $k$ points in the FBZ) was used, where starting from a selfconsistent scalar relativistic calculation, the MAE is evaluated in one-step calculations as the band energy difference between different magnetization directions. In addition, self-consistent relativistic calculations with up to $32 \times 32 \times 32 k$ points in the FBZ were used to check the MAE for hard magnetic alloys identified in this study. Further, the magnetic anisotropy of $\mathrm{Mn}_{3} \operatorname{Ir}(\mathrm{N})$ and $\mathrm{Mn}_{3} \operatorname{Pt}(\mathrm{N})$ in the noncollinear ground state was calculated using the VASP code employing the magnetic force 
theorem. Here, a cutoff energy $E=400 \mathrm{eV}$ and a $k$ mesh of $12 \times 12 \times 12 k$ points in the FBZ were used.

The results have been cross-checked with self-consistent calculations, including spin-orbit coupling using a cutoff energy $E=500 \mathrm{eV}$ and $k$ meshes of $25 \times 25 \times 25 k$ points $\left(\mathrm{Mn}_{3} \mathrm{IrN}\right)$ and $13 \times 13 \times 13 k$ points $\left(\mathrm{Mn}_{3} \mathrm{PtN}\right)$, showing a convergence of the anisotropy energy of about $1 \mathrm{meV}$.

The anomalous Hall conductivity (AHC) is determined by using the WANNIERTOOLS code, which is implemented within the framework of tight-binding (TB) models [55]. The maximally localized Wannier function (MLFW) TB models are obtained by using the WANNIER90 code [56]. We considered the $s, p$, and $d$ orbitals for Mn and Ir atoms, and $s$ plus $p$ orbitals were incorporated for $\mathrm{N}$, resulting in 72 and 80 MLFWs for $\mathrm{Mn}_{3} \mathrm{Ir}$ and $\mathrm{Mn}_{3} \mathrm{IrN}$, respectively. To evaluate the AHC, the Berry curvature integration is carried out using a uniform $k$ point mesh of $401 \times 401 \times 401$, which is expressed as follows [57]:

$$
\begin{array}{r}
\sigma_{x y}=-\frac{e^{2}}{\hbar} \int \frac{d \mathbf{k}}{(2 \pi)^{3}} \sum_{n} f[\epsilon(\mathbf{k})-\mu] \Omega_{n, x y}(\mathbf{k}), \\
\Omega_{n, x y}(\mathbf{k})=-2 \operatorname{Im} \sum_{m \neq n} \frac{\left\langle\psi_{\mathbf{k} n}\left|v_{x}\right| \psi_{\mathbf{k} m}\right\rangle\left\langle\psi_{\mathbf{k} m}\left|v_{y}\right| \psi_{\mathbf{k} n}\right\rangle}{\left[\epsilon_{m}(\mathbf{k})-\epsilon_{n}(\mathbf{k})\right]^{2}},
\end{array}
$$

where $e$ is elementary charge, $\mu$ is the chemical potential, $\psi_{n / m}$ denotes the Bloch wave function with energy eigenvalue $\epsilon_{n / m}, v_{x / y}$ is the velocity operator along the Cartesian $x / y$ direction, and $f[\epsilon(\mathbf{k})-\mu]$ is the Fermi-Dirac distribution function. In this work, the anomalous Nernst coefficient (ANC) $\alpha_{x y}$ is determined by using the Mott relation, where we considered only the derivative of AHC at the Fermi level $d \sigma_{x y} / d \epsilon$, which provides a quantitative measure of the ANC:

$$
\alpha_{x y}=-\left.\frac{\pi^{2} k_{B}^{2} T}{3 e} \frac{d \sigma_{x y}}{d \epsilon}\right|_{\epsilon=\mu} .
$$

Unless otherwise mentioned, the generalized gradient approximation (GGA) in the parametrization of Perdew, Burke, and Ernzerhof [58] was used. For calculations in the local spin density approximation (LSDA), which often provides a better description of the spin magnetic moments for weakly correlated systems, the parametrization of Perdew and Wang [59] is used.

For the detection of suitable interstitial positions, the unit cell is scanned on a discrete mesh (here $24 \times 24 \times 24$ points) for sufficient empty space, taking into account the covalent radii $r_{j}$ of the atoms as tabulated in the atomic simulation environment (ASE) [60,61]. As a condition for suitable positions for the interstitial $I$ we use $d_{\min }=\min _{j}\left[d_{I j}-s\left(r_{I}+r_{j}\right)\right]>0$ for all neighboring atoms $j$, where $s=0.7$ is a scaling factor and $d_{I j}$ is the distance between the interstitial and the atom $j$. This approach is similar to the detection of empty spheres used in some electronic structure codes. Preference can be given to high-symmetry positions, which was done for the present calculations. The resulting interstitial positions for the $\mathrm{Cu}_{3} \mathrm{Au}$ structure are shown in Fig. 2. The interstitial concentration was varied using $2 \times 2 \times 2,1 \times 2 \times 2,1 \times 1 \times 2$, and $1 \times 1 \times 1$ supercells with one interstitial position occupied, corresponding to interstitial concentrations of 3.0, 5.9, 11.1, and 20 at.\%. An additional slight orthorhombic distortion
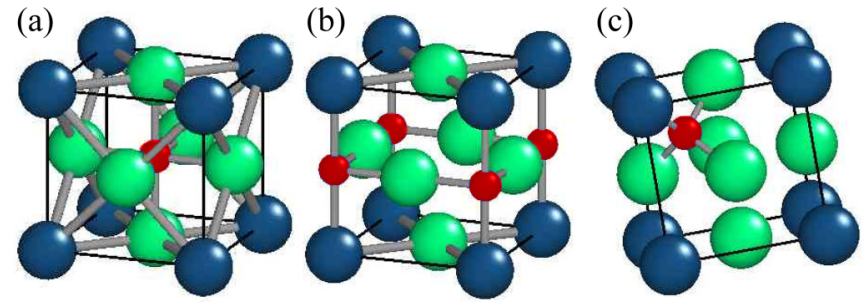

FIG. 2. Interstitial positions in the $\mathrm{Cu}_{3} \mathrm{Au}$ structure: (a) $1 \mathrm{~b}:(1 / 2$, $1 / 2,1 / 2)$; (b) $3 \mathrm{~d}:(0,0,1 / 2)$; and (c) $8 \mathrm{~g}:(x, x, x)$, with $x \approx 3 / 4$. The interstitial positions are highlighted in red.

(2\%-5\% change in the lattice parameters) of the interstitial supercells was also tested but did not have an effect on the stable interstitial compounds discussed in this paper.

The calculation of convex hulls is based on about 2000 alloys (experimental structures and structures close to the convex hull reported in the Materials Project [62]) for ternary $M-X-Z$ systems $(M=\mathrm{Fe}, \mathrm{Co}, \mathrm{Ni}, \mathrm{Cr}$ and $\mathrm{Mn} ; Z=\mathrm{B}, \mathrm{C}, \mathrm{N} ; X$ : other element). For the competing phases, the same numerical setup as for the interstitial compounds was used. For compounds with $\mathrm{Mn}$ atoms, where antiferromagnetic couplings are to be expected, the magnetic ground state was determined for the parent compounds with $\mathrm{Cu}_{3} \mathrm{Au}$ structure and their stable interstitial alloys. To ensure reliable phase diagrams for the Mn systems, the magnetic ground state was also determined for the experimentally reported competing phases and some of the energetically low-lying phases reported in the Materials Project. Depending on the size and symmetry of the cell, we used spin configurations compatible with the maximal magnetic subgroups with propagation vector $\mathbf{q}=$ $(0,0,0),(1 / 2,1 / 2,0),(0,0,1 / 2)$ or, alternatively, an antiferromagnetic coupling of sublattices to probe the magnetic ground state.

In the case of $\mathrm{Mn}_{3}$ Ir with the $\mathrm{Cu}_{3} \mathrm{Au}$ prototype this results (after removal of symmetry-equivalent configurations) in three collinear [ferromagnetic (FM), AF, and ferrimagnetic] and one noncollinear initial spin structures for $\mathbf{q}=(0,0,0)$, which is sufficient to obtain the experimentally reported ground state. It is noteworthy that the experimentally reported magnetic structure of $\mathrm{Mn}_{3}$ Ir does not have maximal magnetic subgroup symmetry but is compatible with the symmetry of the second generation of maximal subgroups [maximal subgroup $R 3 m^{\prime}$ (No. 166.101) of the maximal subgroup $P m \overline{3} m^{\prime}$ (No. 221.95)]. Nevertheless, the NC initial structures relax to the correct ground state, which underpins the high efficiency of the approach. We explicitly checked that the second generation of maximal subgroups results in the same magnetic ground state. The AF coupling of sublattices corresponds to the highest-ranked magnetic structures in the prioritization scheme proposed in Ref. [33] and is especially useful when the paramagnetic structure has a relatively large number of inequivalent magnetic atoms $N_{\mathrm{AF}}$, which are allowed to couple AF. The number of possible magnetic configurations at this prioritization level is $2^{N_{\mathrm{AF}}-1}$, which results, for instance, for $\mathrm{Mn}_{7} \mathrm{C}_{3}$ of the hexagonal $\mathrm{Cr}_{7} \mathrm{C}_{3}$ prototype (with $56 \mathrm{Mn}$ atoms in the unit cell and $N_{\mathrm{AF}}=8$ ), in 128 possible magnetic configurations. 
TABLE I. Properties of parent compounds in the $\mathrm{Cu}_{3} \mathrm{Au}$ structure. Experimental $\left(a_{\mathrm{exp}}\right)$ and calculated $\left(a_{\mathrm{GGA}}\right)$ lattice parameters, the calculated formation energy $E_{f}$ and distance from the convex hull $\Delta E_{h}$, and the total magnetic moment $M$ are shown.

\begin{tabular}{|c|c|c|c|c|c|}
\hline Alloy & $a_{\exp }(\AA)$ & $a_{\mathrm{GGA}}(\AA)$ & $E_{f}(\mathrm{eV} /$ atom $)$ & $\Delta E_{h}(\mathrm{eV} /$ atom $)$ & $M$ (in units of $\mu_{B} /$ f.u.) \\
\hline $\mathrm{Co}_{3} \mathrm{Al}$ & 3.66 & 3.57 & -0.175 & 0.124 & 3.86 \\
\hline $\mathrm{Co}_{3} \mathrm{Ta}$ & 3.65 & 3.63 & -0.241 & 0.001 & 0.02 \\
\hline $\mathrm{Co}_{3} \mathrm{Ti}$ & 3.62 & 3.60 & -0.257 & 0.000 & 2.71 \\
\hline $\mathrm{Fe}_{3} \mathrm{Ga}$ & 3.70 & 3.66 & -0.133 & 0.000 & 6.98 \\
\hline $\mathrm{Fe}_{3} \mathrm{Ge}$ & 3.68 & 3.63 & -0.099 & 0.010 & 6.37 \\
\hline $\mathrm{Fe}_{3} \mathrm{Pt}$ & 3.77 & 3.73 & -0.076 & 0.041 & 8.46 \\
\hline $\mathrm{Fe}_{3} \mathrm{Sn}$ & 3.87 & 3.82 & 0.086 & 0.088 & 6.94 \\
\hline $\mathrm{Mn}_{3} \mathrm{Ir}$ & 3.77 & 3.71 & -0.215 & 0.000 & $0.01^{\mathrm{a}}$ \\
\hline $\mathrm{Mn}_{3} \mathrm{Pt}$ & 3.84 & 3.74 & -0.251 & 0.000 & $0.00^{\mathrm{a}}$ \\
\hline $\mathrm{Mn}_{3} \mathrm{Rh}$ & 3.81 & 3.71 & -0.142 & 0.000 & $0.00^{\mathrm{a}}$ \\
\hline $\mathrm{Mn}_{3} \mathrm{Sb}$ & 4.00 & 3.91 & 0.076 & 0.086 & $0.04^{\mathrm{a}}$ \\
\hline $\mathrm{Ni}_{3} \mathrm{Fe}$ & 3.55 & 3.55 & -0.088 & 0.000 & 4.72 \\
\hline $\mathrm{Ni}_{3} \mathrm{Ga}$ & 3.58 & 3.59 & -0.289 & 0.000 & 0.79 \\
\hline $\mathrm{Ni}_{3} \mathrm{Ge}$ & 3.57 & 3.57 & -0.292 & 0.000 & 0.05 \\
\hline $\mathrm{Ni}_{3} \mathrm{In}$ & 3.75 & 3.75 & -0.052 & 0.045 & 0.69 \\
\hline $\mathrm{Ni}_{3} \mathrm{Mn}$ & 3.59 & 3.56 & -0.102 & 0.000 & 4.85 \\
\hline $\mathrm{Ni}_{3} \mathrm{Si}$ & 3.51 & 3.51 & -0.462 & 0.000 & 0.04 \\
\hline $\mathrm{Ni}_{3} \mathrm{Sn}$ & 3.74 & 3.74 & -0.188 & 0.000 & 0.06 \\
\hline $\mathrm{Pt}_{3} \mathrm{Co}$ & 3.83 & 3.88 & -0.061 & 0.000 & 2.91 \\
\hline $\mathrm{Pt}_{3} \mathrm{Cr}$ & 3.88 & 3.91 & -0.254 & 0.000 & 2.60 \\
\hline $\mathrm{Rh}_{3} \mathrm{Cr}$ & 3.79 & 3.78 & -0.122 & 0.000 & 1.24 \\
\hline
\end{tabular}

${ }^{\mathrm{a}}$ Noncollinear magnetic structure.

While this approach does not ensure that the correct magnetic ground state is found in all cases, we believe it should give a reliable basis for the prediction of the phase stability in these systems. For the remaining alloys a ferromagnetic magnetic configuration was assumed.

\section{RESULTS OF HIGH-THROUGHPUT SCREENING}

\section{A. Parent compounds in the $\mathrm{Cu}_{3} \mathrm{Au}$ structure}

Table I shows the calculated properties of the 21 parent compounds with $\mathrm{Cu}_{3} \mathrm{Au}$ structure. The calculated lattice parameters are in good agreement with the reported experimental values, with the largest deviation of about $2.6 \%$ for $\mathrm{Mn}_{3} \mathrm{Rh}$ and $\mathrm{Mn}_{3} \mathrm{Pt}$. With the exception of $\mathrm{Mn}_{3} \mathrm{Sb}$ and $\mathrm{Fe}_{3} \mathrm{Sn}$ the calculated formation energy is negative for all compounds, as expected from the formation of alloys in nature. $\mathrm{Mn}_{3} \mathrm{Sb}$ was only recently synthesized by high-pressure synthesis [63], and the $\mathrm{Cu}_{3} \mathrm{Au}$ structure of $\mathrm{Fe}_{3} \mathrm{Sn}$ was reported in high-pressure studies, whereas the ambient pressure phase is hexagonal, in agreement with our calculations (see the Supplemental Material [64]). For 18 of the 21 compounds the calculated distance from the convex hull $\Delta E_{h}$ is below $50 \mathrm{meV} /$ atom, which was proposed in Ref. [51] as a simple descriptor for potentially stable or metastable alloys in high-throughput calculations. In addition to the above-mentioned $\mathrm{Mn}_{3} \mathrm{Sb}$ and $\mathrm{Fe}_{3} \mathrm{Sn}$ alloys, only $\mathrm{Co}_{3} \mathrm{Al}$ is above this criterion, which shows the largest $\Delta E_{h}=124 \mathrm{meV} /$ atom among the calculated compounds. A $\mathrm{Cu}_{3} \mathrm{Au}$ structure of $\mathrm{Co}_{3} \mathrm{Al}$ was discussed in the literature but could not be stabilized experimentally [65]. This result is confirmed by our study, where only the Al-rich phases AlCo, $\mathrm{Al}_{5} \mathrm{Co}_{2}, \mathrm{Al}_{13} \mathrm{Co}_{4}$, and $\mathrm{Al}_{9} \mathrm{Co}_{2}$ are found to be stable. A complete list of the alloys relevant for the calculation of the convex hull in Table $\mathrm{I}$ is given in the Supplemental Material [64].

For $\mathrm{Ni}_{3} X(X=\mathrm{Si}, \mathrm{Ge}, \mathrm{Sn})$ and $\mathrm{Co}_{3} \mathrm{Ta}$ the Fermi energy lies in a pseudogap or valley of the density of states (DOS; see Fig. 3), resulting in a nonmagnetic or only weakly ferromagnetic ground state despite the presence of the magnetic elements $\mathrm{Ni}$ and Co. For $\mathrm{Mn}_{3} X(X=\mathrm{Rh}, \mathrm{Ir}, \mathrm{Pt}$, and $\mathrm{Sb})$ the calculated ground state is noncollinear. The antiferromagnetic coupling of the neighboring Mn atoms cannot be fully satisfied in the $\mathrm{Cu}_{3} \mathrm{Au}$ structure, resulting in a frustrated triangular spin structure with compensated magnetic moments; see, for instance, Ref. [42] for a more detailed discussion. For $X=$ $\mathrm{Rh}, \mathrm{Ir}, \mathrm{Pt}$ this result is in agreement with experiment $[66,67]$ and earlier calculations [42]. In the case of $\mathrm{Mn}_{3} \mathrm{Pt}$ a transition from a triangular to a collinear AF magnetic structure with a

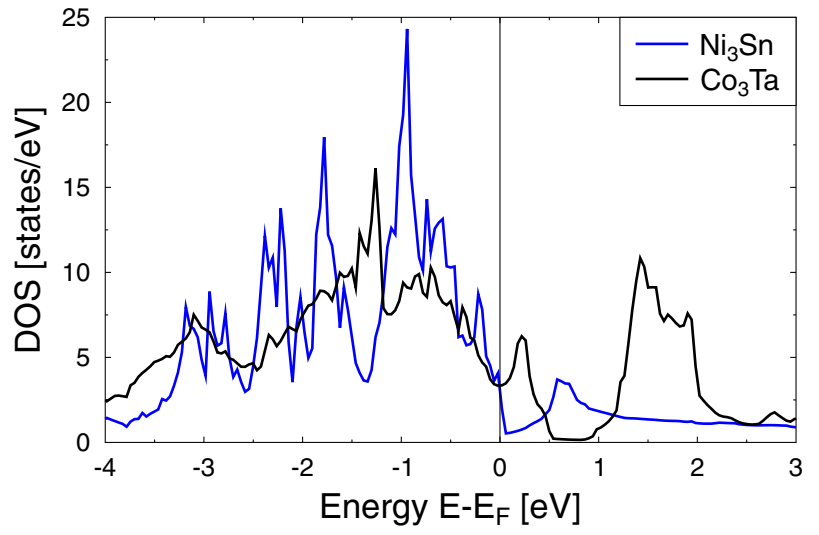

FIG. 3. Total DOS of $\mathrm{Co}_{3} \mathrm{Ta}$ and $\mathrm{Ni}_{3} \mathrm{Sn}$ for the experimental lattice parameters calculated with FPLO using GGA. 
(a)

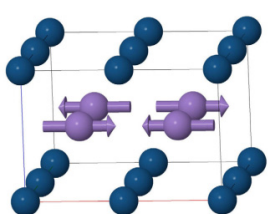

(b)

(c)

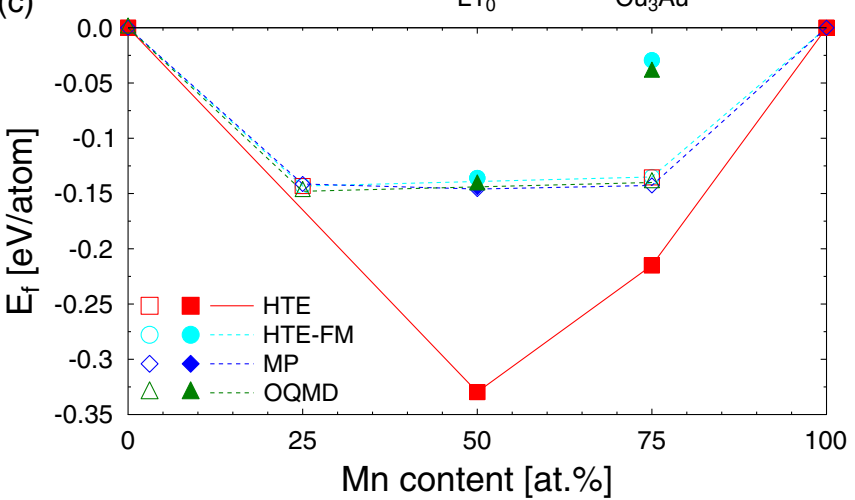

FIG. 4. Calculated magnetic ground-state structures for (a) MnIr and (b) $\mathrm{Mn}_{3}$ Ir. Note that the spin-quantization axis has been rotated to match the results of calculations with SOC. (c) Calculated formation energies for experimental (solid symbols) and hypothetical (open symbols) lattice structures of Mn-Ir alloys for (i) the magnetic ground states (HTE) and (ii) assumed ferromagnetic structures: our calculations (HTE-FM) and data from the Materials Project (MP) and the Open Quantum Materials Database (OQMD). Convex hulls (lines) connect stable structures for the respective methods.

doubled unit cell along $c$ was observed around $365 \mathrm{~K}$ in neutron scattering experiments [66], while a similar transition was not observed for $\mathrm{Mn}_{3} \mathrm{Rh}$ and $\mathrm{Mn}_{3} \mathrm{Ir}$. For $\mathrm{Mn}_{3} \mathrm{Sb}$, the energies of $\mathrm{NC}$, ferrimagnetic, and FM magnetic structures are within less than $35 \mathrm{meV} /$ atom. Experimentally, a collinear magnetic structure with almost compensating Mn magnetic moments was deduced from neutron scattering experiments [68], while more recent Mössbauer studies suggest a triangular magnetic structure [69].

The energy difference $\Delta E_{\mathrm{FM}}$ between the magnetic ground state and a ferromagnetic state can be as large as $243 \mathrm{meV} /$ atom for $\mathrm{Mn}_{3} \mathrm{Pt}$. For some of the competing phases the calculated $\Delta E_{\mathrm{FM}}$ is of similar magnitude, while $\Delta E_{\mathrm{FM}}$ is trivially zero for compounds with a ferromagnetic ground state. The magnetic structure can thus have a deep impact on the calculated phase stability, going beyond typical effects due to finite temperature. This is exemplarily shown in Fig. 4 for binary Mn-Ir alloys. Assuming a ferromagnetic spin alignment, a hexagonal $\mathrm{MnIr}_{3}$ phase, $L 1_{0} \mathrm{MnIr}$, and a hexagonal $\mathrm{Mn}_{3}$ Ir phase are on or nearly on the convex hull, in good agreement with results obtained from the Materials Project [62] and the Open Quantum Materials Database [70]. In contrast, calculations with the magnetic ground state yield the experimentally observed $L 1_{0} \mathrm{MnIr}$ and $\mathrm{Cu}_{3} \mathrm{Au}$ structure of $\mathrm{Mn}_{3} \mathrm{Ir}$ as stable phases on the convex hull. For $L 1_{0} \mathrm{MnIr}$ and $\mathrm{Cu}_{3} \mathrm{Au}-\mathrm{Mn}_{3}$ Ir the magnetic ground-state search resulted in a collinear AF and a NC triangular AF structure, respectively, in agreement with experiment [67,71]. This is accompanied by a significant lowering of the formation energy of nearly $0.2 \mathrm{meV} /$ atom. In contrast, the magnetic ground-state search
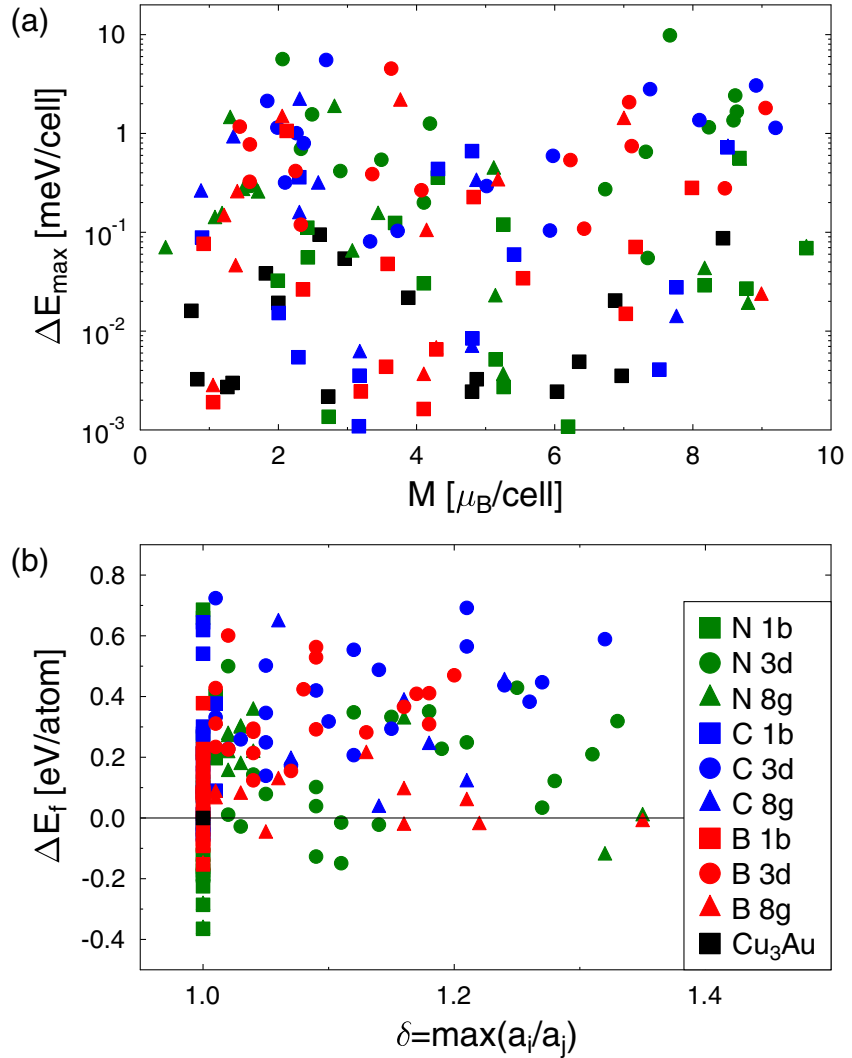

FIG. 5. (a) Maximum energy difference $\Delta E_{\max }=\max \left(E_{i}-E_{j}\right)$ between different magnetization directions versus total magnetic moment $M$. (b) Maximal change in the ratio of lattice parameters $\delta=\max \left(a_{i} / a_{j}\right)$ versus change in the formation energy $\Delta E_{f}$. Data are shown for parent compounds in the $\mathrm{Cu}_{3} \mathrm{Au}$ structure (solid black squares) and interstitial structures with $\mathrm{B}$ (red), $\mathrm{C}$ (blue), and $\mathrm{N}$ (green). The interstitial $1 \mathrm{~b}, 3 \mathrm{~d}$, and $8 \mathrm{~g}$ positions are marked with solid squares, circles, and triangles, respectively.

for the two (experimentally not observed) hexagonal phases did not have an effect on the calculated formation energy. An impact of the magnetic structure on the calculated phase stability similar to that for $\mathrm{Mn}-\mathrm{Ir}$ is found also in other Mn alloy systems and will be discussed in more detail elsewhere.

\section{B. Effect of interstitials on structural and magnetic properties}

Figure 5(a) shows the calculated MAE (maximum energy difference between magnetization directions [001], [010], [100], [110], and [111]) for the $\mathrm{Cu}_{3} \mathrm{Au}$ parent compounds and interstitial alloys with one $\mathrm{N}, \mathrm{C}$, or $\mathrm{B}$ atom per f.u., assuming a ferromagnetic alignment of the spins. It can be seen that interstitials can induce huge MAEs up to about $2 \mathrm{meV} /$ atom, one to two orders higher in magnitude than in their cubic parent compounds. N, C, and B are similarly efficient in inducing these large MAEs. The largest MAEs are calculated for the $3 d$ interstitial position, which leads to a tetragonal distortion of the lattice and therefore is promising to achieve uniaxial MAEs. There can be also a significant MAE between the [001], [110], and [111] directions of up to about $0.2 \mathrm{meV} /$ atom for the cubic compounds with heavy $5 d$ 
TABLE II. Interstitial alloys with a distance from the convex hull $\Delta E_{h}<50 \mathrm{meV} /$ atom. The parent compound with the $\mathrm{Cu}_{3} \mathrm{Au}$ structure, the interstitial atom and position, and the highest interstitial concentration $c_{\max }$ with $\Delta E_{h}<50 \mathrm{meV} /$ atom (with three different values of the interstitial concentrations: $5.9,11.1$, and 20) in atomic percent are shown along with the calculated formation energy $E_{f}, \Delta E_{h}$, and the total magnetic moment $M$ per atom.

\begin{tabular}{|c|c|c|c|c|c|}
\hline Parent & Interstitial & $c_{\max }$ & $E_{f}(\mathrm{eV} /$ atom $)$ & $\Delta E_{h}(\mathrm{eV} /$ atom $)$ & $M$ (in units of $\mu_{B} /$ atom) \\
\hline $\mathrm{Co}_{3} \mathrm{Al}$ & $\mathrm{C} 1 b$ & 20.0 & -0.230 & 0.010 & 0.00 \\
\hline $\mathrm{Co}_{3} \mathrm{Ta}$ & $\mathrm{B} 1 b$ & 11.1 & -0.272 & 0.044 & 0.00 \\
\hline $\mathrm{Co}_{3} \mathrm{Ta}$ & $\mathrm{C} 1 b$ & 11.1 & -0.211 & 0.035 & 0.00 \\
\hline $\mathrm{Co}_{3} \mathrm{Ti}$ & В $1 b$ & 11.1 & -0.324 & 0.043 & 0.31 \\
\hline $\mathrm{Co}_{3} \mathrm{Ti}$ & $\mathrm{C} 1 b$ & 11.1 & -0.302 & 0.003 & 0.22 \\
\hline $\mathrm{Fe}_{3} \mathrm{Ga}$ & B $1 b$ & 5.9 & -0.124 & 0.047 & 1.50 \\
\hline $\mathrm{Fe}_{3} \mathrm{Ga}$ & $\mathrm{C} 1 b$ & 20.0 & -0.063 & 0.044 & 0.63 \\
\hline $\mathrm{Fe}_{3} \mathrm{Ga}$ & $\mathrm{N} 1 b$ & 20.0 & -0.255 & 0.016 & 1.05 \\
\hline $\mathrm{Fe}_{3} \mathrm{Ge}$ & $\mathrm{N} 1 b$ & 5.9 & -0.096 & 0.040 & $1.32^{\mathrm{a}}$ \\
\hline $\mathrm{Fe}_{3} \mathrm{Pt}$ & $\mathrm{N} 1 b$ & 20.0 & -0.263 & 0.000 & 1.63 \\
\hline $\mathrm{Fe}_{3} \mathrm{Sn}$ & $\mathrm{N} 1 b$ & 20.0 & -0.104 & 0.037 & 1.02 \\
\hline $\mathrm{Mn}_{3} \mathrm{Ir}$ & $\mathrm{N} 1 b$ & 20.0 & -0.359 & 0.026 & $0.00^{\mathrm{b}}$ \\
\hline $\mathrm{Mn}_{3} \mathrm{Pt}$ & $\mathrm{N} 1 b$ & 20.0 & -0.471 & 0.000 & $0.00^{\mathrm{b}}$ \\
\hline $\mathrm{Mn}_{3} \mathrm{Rh}$ & $\mathrm{N} 1 b$ & 20.0 & -0.356 & 0.000 & $0.00^{\mathrm{b}}$ \\
\hline $\mathrm{Mn}_{3} \mathrm{Sb}$ & $\mathrm{N} 1 b$ & 20.0 & -0.226 & 0.036 & $0.55^{\mathrm{a}}$ \\
\hline $\mathrm{Ni}_{3} \mathrm{Fe}$ & $\mathrm{N} 3 d$ & 5.9 & -0.063 & 0.038 & 1.05 \\
\hline $\mathrm{Ni}_{3} \mathrm{Ga}$ & B $1 b$ & 5.9 & -0.305 & 0.014 & 0.00 \\
\hline $\mathrm{Ni}_{3} \mathrm{Ga}$ & C $1 b$ & 5.9 & -0.273 & 0.006 & 0.00 \\
\hline $\mathrm{Ni}_{3} \mathrm{Ga}$ & $\mathrm{N} 1 b$ & 11.1 & -0.258 & 0.036 & 0.00 \\
\hline $\mathrm{Ni}_{3} \mathrm{In}$ & В $1 b$ & 20.0 & -0.205 & 0.049 & 0.00 \\
\hline $\mathrm{Ni}_{3} \mathrm{In}$ & C $1 b$ & 20.0 & -0.040 & 0.038 & 0.00 \\
\hline $\mathrm{Ni}_{3} \mathrm{In}$ & $\mathrm{N} 1 b$ & 20.0 & -0.173 & 0.000 & 0.00 \\
\hline $\mathrm{Ni}_{3} \mathrm{Mn}$ & В $1 b$ & 5.9 & -0.110 & 0.041 & 1.09 \\
\hline $\mathrm{Ni}_{3} \mathrm{Mn}$ & C $1 b$ & 5.9 & -0.071 & 0.025 & 1.08 \\
\hline $\mathrm{Ni}_{3} \mathrm{Mn}$ & $\mathrm{N} 3 d$ & 11.1 & -0.113 & 0.044 & 0.95 \\
\hline $\mathrm{Ni}_{3} \mathrm{Sn}$ & В $1 b$ & 5.9 & -0.196 & 0.038 & 0.00 \\
\hline $\mathrm{Ni}_{3} \mathrm{Sn}$ & $\mathrm{C} 1 b$ & 5.9 & -0.134 & 0.043 & 0.00 \\
\hline $\mathrm{Ni}_{3} \mathrm{Sn}$ & $\mathrm{N} 1 b$ & 11.1 & -0.135 & 0.045 & 0.00 \\
\hline $\mathrm{Pt}_{3} \mathrm{Co}$ & B $8 g$ & 11.1 & -0.069 & 0.048 & 0.59 \\
\hline
\end{tabular}

${ }^{\mathrm{a}}$ Antiferromagnetic or ferrimagnetic structure.

${ }^{\mathrm{b}}$ Noncollinear magnetic structure.

elements (while the energy difference between [001], [010], and [100] is zero due to symmetry). It should be noted, however, that neither stability nor potential antiferromagnetic spin configurations are considered in this plot. The MAEs shown in Fig. 5 thus rather serve to illustrate the potential of interstitials in inducing large MAEs, while for the majority of the data points an experimental realization will not be possible.

This becomes also clear from Fig. 5(b), which shows the formation energy difference $\Delta E_{f}$ with respect to the $\mathrm{Cu}_{3} \mathrm{Au}$ parent compound in relation to the induced structural distortion for different interstitial atoms and positions. For the majority of data points $\Delta E_{f}$ is positive, indicating that the interstitial alloy is unlikely to form, at least at the interstitial concentration of 20 at.\% considered in the plot. Further, in most cases the $1 b$ interstitial position, which does not break the cubic symmetry, is the most stable. In most cases this means that a significant enhancement of the MAE is not expected. Noteworthy exceptions, where the spin structure breaks the cubic symmetry, will be discussed further below. Significant structural distortions are found for the $3 d$ and $8 g$ interstitial positions with negative $\Delta E_{f}$ in some cases, suggesting that the interstitial alloys are likely to form. For the $8 g$ interstitial position, which is least favorable from geometrical considerations, we often find relaxation to a structure corresponding to the $1 b$ or $3 d$ position, which provides a higher volume for the interstitial atom.

\section{Stable interstitial phases}

Table II lists interstitial alloys, where the distance to the convex hull $\Delta E_{h}$ is below $50 \mathrm{meV} /$ atom for an interstitial concentration of more than 5 at.\%, suggesting that a substantial number of interstitial atoms can be incorporated in the parent alloy with $\mathrm{Cu}_{3} \mathrm{Au}$ structure. This criterion is matched for 29 parent-interstitial combinations, including known antiperovskites like $\mathrm{Mn}_{3} X \mathrm{~N}(X=\mathrm{Rh}, \mathrm{Ir}, \mathrm{Pt}$, and $\mathrm{Sb})$ [45,72], $\mathrm{Ni}_{3} \mathrm{InN}$ [73], $\mathrm{Fe}_{3} \mathrm{PtN}$ [74], $\mathrm{Co}_{3} \mathrm{AlC}$ [75], $\mathrm{Fe}_{3} \mathrm{GaN}$ [76], and $\mathrm{Fe}_{3} \mathrm{SnN}$ [77]. Only the most stable interstitial position is listed. In some cases more than one interstitial position lowers the formation energy, suggesting a partial occupation of the interstitial sites. This is, for instance, the case for $\mathrm{Ni}_{3} \mathrm{Mn}$, where the $\mathrm{N} 3 d$ and $1 b$ positions are close in energy and both of them fulfill the $\Delta E_{h}$ criterion for 5.9 at. $\% \mathrm{~N}$ concentration. 
TABLE III. Stable alloys with large uniaxial $\mathrm{MAE}=E_{[001]}-$ $E_{[100]}$. The total magnetic spin moment $M$ and the MAE calculated within GGA and LSDA are listed. A collinear spin arrangement is assumed.

\begin{tabular}{lccc}
\hline \hline Alloy & Interstitial & $\begin{array}{c}M_{\mathrm{GGA}} / M_{\mathrm{LSDA}} \\
\text { (in units of } \mu_{B} / \text { atom) }\end{array}$ & $\begin{array}{c}\mathrm{MAE}_{\mathrm{GGA}} / \mathrm{MAE}_{\mathrm{LSDA}} \\
(\mathrm{meV} / \text { atom) }\end{array}$ \\
\hline $\mathrm{Mn}{ }_{3} \mathrm{Ir}$ & & $0.37^{\mathrm{a}} / 0.30^{\mathrm{a}}$ & $-0.19 /-0.43$ \\
$\mathrm{Mn}_{3} \mathrm{IrN}$ & $\mathrm{N} 1 b$ & $0.54^{\mathrm{a}} / 0.49^{\mathrm{a}}$ & $-1.32 /-1.27$ \\
$\mathrm{Mn}{ }_{3} \mathrm{Pt}$ & & $0.36^{\mathrm{a}} / 0.32^{\mathrm{a}}$ & $-0.09 /-0.11$ \\
$\mathrm{Mn}_{3} \mathrm{PtN}$ & $\mathrm{N} 1 b$ & $0.63^{\mathrm{a}} / 0.57^{\mathrm{a}}$ & $-0.16 /-0.22$ \\
$\mathrm{Mn}_{3} \mathrm{Rh}$ & & $0.43^{\mathrm{a}} / 0.37^{\mathrm{a}}$ & $-0.10 /-0.12$ \\
$\mathrm{Mn}_{3} \mathrm{RhN}$ & $\mathrm{N} 1 b$ & $0.56^{\mathrm{a}} / 0.51^{\mathrm{a}}$ & $-0.31 /-0.29$ \\
$\mathrm{Mn}_{3} \mathrm{SbN}$ & $\mathrm{N} 1 b$ & $0.55^{\mathrm{a}} / 0.52^{\mathrm{a}}$ & $-0.20 /-0.13$ \\
$\mathrm{Ni}_{3} \mathrm{MnN}_{0.5}$ & $\mathrm{~N} 3 d$ & $0.96 / 0.90$ & $-0.09 /-0.10$ \\
\hline \hline
\end{tabular}

${ }^{\mathrm{a}}$ Compounds with a ferrimagnetic structure.

The largest gain in formation energy with respect to the parent compound $\Delta E_{f} \approx-0.3 \mathrm{eV} /$ atom is found for $\mathrm{Mn}_{3} \mathrm{SbN}$, where the "interstitial" alloy [72] was reported more than 20 years before the metastable "parent" alloy $\mathrm{Mn}_{3} \mathrm{Sb}$ [63]. For the majority of interstitial alloys, stability is accompanied by a negative $\Delta E_{f}$. However, even a large negative $\Delta E_{f}$ does not ensure formation of a stable alloy. This is the case, for instance, for $\mathrm{Co}_{3} \mathrm{AlN}$ and $\mathrm{Co}_{3} \mathrm{TiN}$, where the distance from the convex hull is $0.3-0.4 \mathrm{eV} /$ atom, despite $\Delta E_{f}$ values of about -0.16 and $-0.13 \mathrm{eV} /$ atom, respectively. Conversely, a positive $\Delta E_{f}$ does not prevent the formation of a stable interstitial alloy, as in the case of $\mathrm{Fe}_{3} \mathrm{GaC}_{x}$.

For the majority of interstitials, the $1 b$ position is energetically preferred. The $3 d$ position, which is especially interesting for hard magnetic applications as it breaks the cubic symmetry and leads to a tetragonal distortion of the lattice, is energetically most preferable for $\mathrm{Ni}_{3} \mathrm{FeN}_{x}, \mathrm{Ni}_{3} \mathrm{MnN}_{x}$, $\mathrm{Co}_{3} \mathrm{TaN}_{x}$, and $\mathrm{Co}_{3} \mathrm{TiN}_{x}$. In all cases substantial MAEs between -0.67 and $1.3 \mathrm{meV} /$ cell are induced for $x=1$ (20 at. $\%$ $\mathrm{N})$. The largest absolute value of $1.3 \mathrm{meV} /$ cell is found for $\mathrm{Ni}_{3} \mathrm{MnN}$, albeit with an easy-plane magnetization. However, for $\mathrm{Co}_{3} \mathrm{TaN}_{x}$ and $\mathrm{Co}_{3} \mathrm{TiN}_{x}$ only a minor number of interstitials is expected to be incorporated due to the formation of extraordinary stable Ta-N and Ti-N phases (see the Supplemental Material [64]). For realistic $\mathrm{N}$ concentrations (11 at.\% $\mathrm{N}$, $x=0.5), \mathrm{Ni}_{3} \mathrm{MnN}_{x}$ shows a significant uniaxial MAE around $-100 \mu \mathrm{eV} /$ atom, comparable to hcp Co. For $\mathrm{Ni}_{3} \mathrm{FeN}_{x}$, the MAE is small ( $\approx 15 \mu \mathrm{eV} /$ atom $)$ and depends on the choice of supercell.

Table III lists stable alloys with large uniaxial MAE for the most stable collinear magnetic structure obtained in the preceding VASP calculations. For comparison, results obtained within LSDA (employing the same GGA optimized structure) are also shown. For both GGA and LSDA, the listed alloys show a large uniaxial MAE, but the absolute values can differ significantly. While GGA typically yields lattice parameters in better agreement with experiment, the local spin magnetic moments are often better described within LSDA. As the MAE depends on subtle details of the electronic structure, this can have a strong impact on the calculated MAE [78], as is the case here for $\mathrm{Mn}_{3} \mathrm{Ir}, \mathrm{Mn}_{3} \mathrm{PtN}$, and $\mathrm{Mn}_{3} \mathrm{SbN}$. According to Table III, $\mathrm{Mn}_{3} X \mathrm{~N}$ and $\mathrm{Mn}_{3} X$ (X $=\mathrm{Rh}, \mathrm{Ir}, \mathrm{Pt}$, and $\mathrm{Sb}$ ) would have a huge potential for application as hard magnets, provided they could be stabilized in a ferrimagnetic structure. The largest MAE of about $-1.3 \mathrm{meV} /$ atom is obtained for $\mathrm{Mn}_{3} \mathrm{IrN}$. A comparison with Tables I and II shows, however, that with the exception of $\mathrm{Mn}_{3} \mathrm{SbN}$ these large MAEs cannot be readily used in bulk magnets, as the ground state is noncollinear with zero net magnetization. However, the strong MAE could be used in exchange-coupled multilayer structures, where a hard magnetic antiferromagnetic layer is coupled to a soft magnetic layer with large magnetization and high Curie temperature. Moreover, as $\mathrm{Mn}_{3} \mathrm{SbN}$ shows, a ferrimagnetic ground state may be induced either by variation of the interstitial concentration or epitaxial strain in a thin film. This will be further explored in Sec. IV.

A comparison of Tables I and II shows some remarkable effects of the interstitials on the magnetic properties. While the ground state of $\mathrm{Co}_{3} \mathrm{Al}$ is ferromagnetic, $\mathrm{Co}_{3} \mathrm{AlC}$ is found to be nonmagnetic. Similarly, the total magnetic moment of $\mathrm{Co}_{3} \mathrm{Ti}$ is strongly reduced by interstitial $\mathrm{B}$, resulting in a nonmagnetic ground state for (unstable) $\mathrm{Co}_{3} \mathrm{TiB}$. On the contrary, $\mathrm{Co}_{3} \mathrm{Ta}$ is nonmagnetic, while $\mathrm{Co}_{3} \mathrm{TaN}$ would be magnetic if it could be synthesized. This shows that interstitials can be very efficient to tune magnetic transitions, which makes interstitial alloying interesting also for magnetocaloric applications.

\section{DISCUSSION}

In the previous section we showed that interstitial atoms can have a strong impact on the stability, magnetic properties, and MAE of compounds. While in principle huge MAEs are possible (see Fig. 5), stable compounds with the necessary properties for a good permanent magnet are rare, in line with experimental experience. Among the 63 parentinterstitial combinations investigated in this study, $\mathrm{Ni}_{3} \mathrm{MnN}_{x}$ with $x \approx 0.5$ was identified as a stable ferromagnetic alloy with magnetic properties comparable to hcp Co at reduced cost. Further, $\mathrm{Mn}_{3} \mathrm{SbN}$ was identified as a ferrimagnet with large MAE, in agreement with the large magnetostriction observed experimentally in this compound [45]. It can be expected that an extended screening of more parent-interstitial combinations would lead to even more promising candidates for hard magnetic applications.

The most striking observation in this study is, however, the huge MAE in $\mathrm{Mn}_{3} X \mathrm{~N}_{x}(X=\mathrm{Rh}, \mathrm{Ir}, \mathrm{Pt}$, and $\mathrm{Sb})$ and the strong impact of interstitial $\mathrm{N}$ on the MAE in these compounds, especially for $\mathrm{Mn}_{3} \mathrm{IrN}$. Figure 6(a) shows the calculated DOS of $\mathrm{Mn}_{3} \mathrm{IrN}$ and $\mathrm{Mn}_{3} \mathrm{Ir}$. Interstitial $\mathrm{N}$ induces changes in the electronic structure in the range of a few hundred meV, larger than the changes in the electronic structure due to a change in the magnetization direction from the easy [001] axis to the hard [100] axis due to spin-orbit coupling, also shown for comparison in Fig. 6(b). The changes in the electronic structure lead also to a change in the orbital moment anisotropy of $\operatorname{Ir}\left(\Delta \mu_{l}=0.046 \mu_{B}\right.$ for $\mathrm{Mn}_{3} \operatorname{Ir} ; \Delta \mu_{l}=0.073 \mu_{B}$ for $\left.\mathrm{Mn}_{3} \operatorname{IrN}\right)$ and $\operatorname{Mn}\left(\Delta \mu_{l}=0.052 \mu_{B}\right.$ for $\operatorname{Mn}_{3} \operatorname{Ir} ; \Delta \mu_{l}=-0.085 \mu_{B}$ for $\mathrm{Mn}_{3} \mathrm{IrN}$ ), which is closely related to the MAE. Thus, interstitial $\mathrm{N}$ has an impact on the electronic structure and MAE going far beyond structural distortions, in agreement with earlier investigations [36]. 

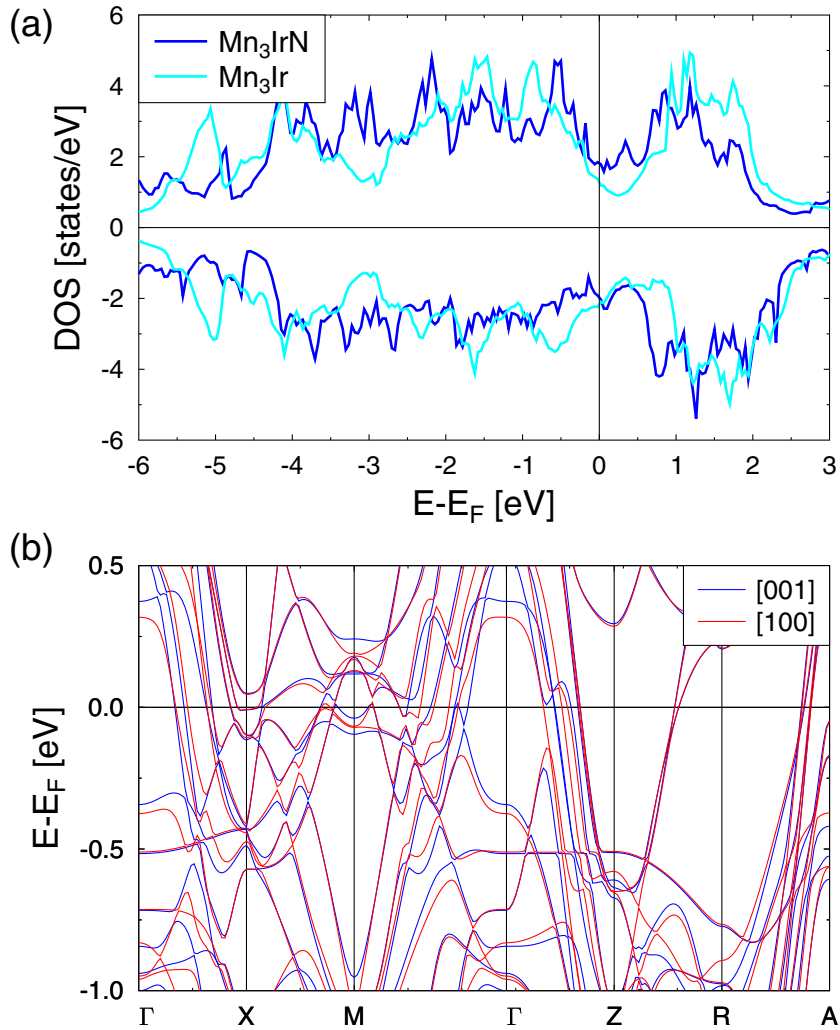

FIG. 6. (a) Comparison of the DOS of ferrimagnetic $\mathrm{Mn}_{3} \mathrm{IrN}$ (blue) and ferrimagnetic $\mathrm{Mn}_{3} \mathrm{Ir}$ (cyan) for the easy [001] axis. (b) Band structure of ferrimagnetic $\mathrm{Mn}_{3} \mathrm{IrN}$ for the easy [001] axis (blue) and the hard [100] axis (red). For simplicity $c / a$ in both graphs was set to 1 .

Another important observation is that according to our calculations, the $\mathrm{N}$ concentration in $\mathrm{Mn}_{3} X \mathrm{~N}_{x}(X=\mathrm{Rh}$, Ir, $\mathrm{Pt}$ ) can be varied in the range between 0 and 20 at.\%, thus providing an additional tuning parameter for the MAE (see Fig. 7). In all cases the interstitial alloys are on or close to the convex hull, well below the $\Delta E_{h}<50 \mathrm{meV} /$ atom criterion for (meta)stability. However, a triangular spin structure is energetically preferred over the collinear ferrimag-

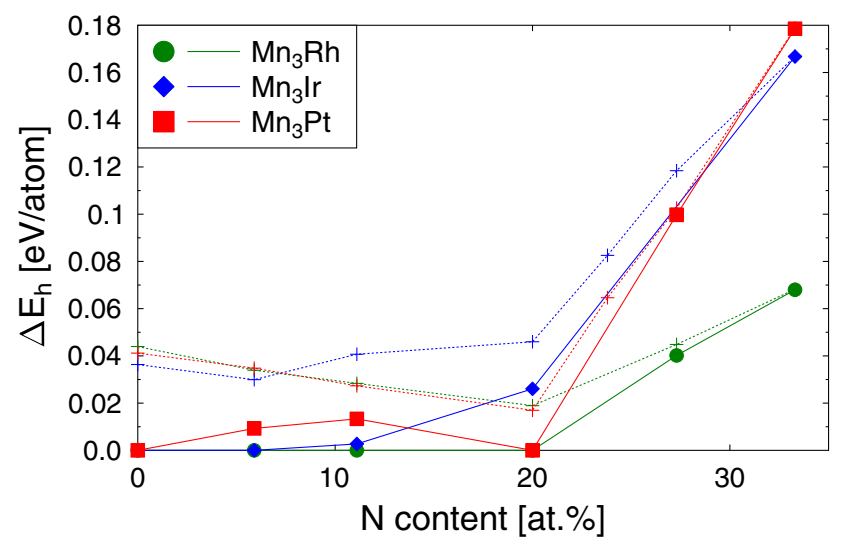

FIG. 7. Distance from the convex hull $\Delta E_{h}$ versus $\mathrm{N}$ concentration for $\mathrm{Mn}_{3} X \mathrm{~N}_{x}(X=\mathrm{Rh}, \mathrm{Ir}, \mathrm{Pt})$ (solid symbols: NC, pluses: ferrimagnetic).

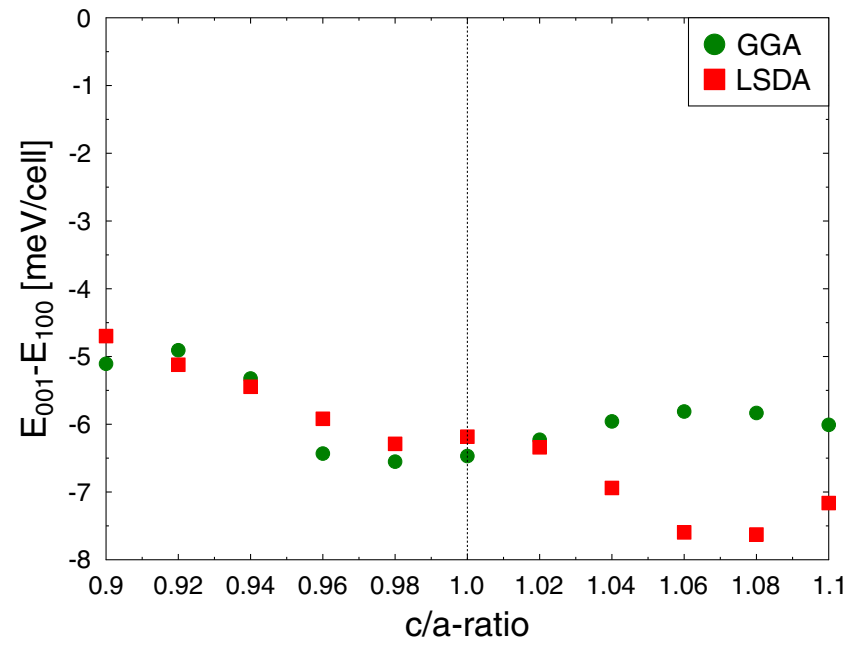

FIG. 8. Calculated MAE versus $c / a$ ratio for $\mathrm{Mn}_{3}$ IrN. Results are shown for GGA (solid green circles) and LSDA (solid red squares). The volume was fixed to the GGA optimized bulk value.

netic structure throughout the whole concentration range. For $\mathrm{Mn}_{3} \mathrm{RhN}_{x}$, where the $\mathrm{N} 1 b$ and $3 d$ positions lead to a gain in formation energy, up to about 30 at.\% interstitial $\mathrm{N}$ can be incorporated into the structure based on the $\Delta E_{h}$ criterion. For these high-N concentrations a ferrimagnetic structure with significant net magnetization around $2 \mu_{B} /$ f.u. and MAE around $-1 \mathrm{meV} / \mathrm{f}$.u. is expected based on our calculations. A partial occupation of the $\mathrm{N} 1 b$ and $3 d$ interstitial positions may also explain deviations from an ideal triangular spin structure reported in this compound [72].

Figure 8 shows the calculated MAE as a function of $c / a$ for $\mathrm{Mn}_{3} \mathrm{IrN}$ in the ferrimagnetic spin configuration employing self-consistent calculations with an enhanced $k$ mesh. First, we note that the MAE obtained from self-consistent calculations for the bulk value is in good agreement with the MAE obtained with the force theorem in the high-throughput calculations, underlining the suitability of the band anisotropy as a descriptor in high-throughput calculations. The results show that the huge MAE is robust against variations of the $c / a$ ratio in epitaxially grown films, with values between -4 and $-8 \mathrm{meV} /$ cell for a wide range of lattice parameters. In particular, the results show that the symmetry breaking due to the ferrimagnetic spin arrangement is responsible for the huge MAE and not a slight distortion of the lattice from cubic symmetry $(c / a=1)$. The total spin moment ranges between $2 \mu_{B}$ and $4 \mu_{B} /$ cell for the $c / a$ ratios investigated. Thus, $\mathrm{Mn}_{3} \mathrm{IrN}$ would be an excellent hard magnetic material if it could be stabilized in the ferrimagnetic spin configuration, which would outperform the known hard magnet $L 1_{0} \mathrm{CoPt}$ with an MAE of about $0.5 \mathrm{meV} /$ atom and a total magnetic moment of about $1.1 \mu_{B} /$ atom. According to our VASP calculations, $\mathrm{N}$ also has a strong impact on the magnetic anisotropy in the $\mathrm{NC}$ ground state and enhances the energy difference $\Delta E=E_{\Gamma^{4 g}}-E_{\Gamma^{5 g}}$ between the easy $\Gamma^{4 g}$ ground state and the $\Gamma^{5 g}$ representation of the magnetic structure from about $-8 \mathrm{meV}\left(\mathrm{Mn}_{3} \mathrm{Ir}\right)$ to $-12 \mathrm{meV}\left(\mathrm{Mn}_{3} \operatorname{IrN}\right)$. Based on these results we expect that interstitial $\mathrm{N}$ also has a strong impact on amorphous $\mathrm{Mn}_{3} \mathrm{Ir}$, which is one of the state-of-the-art 


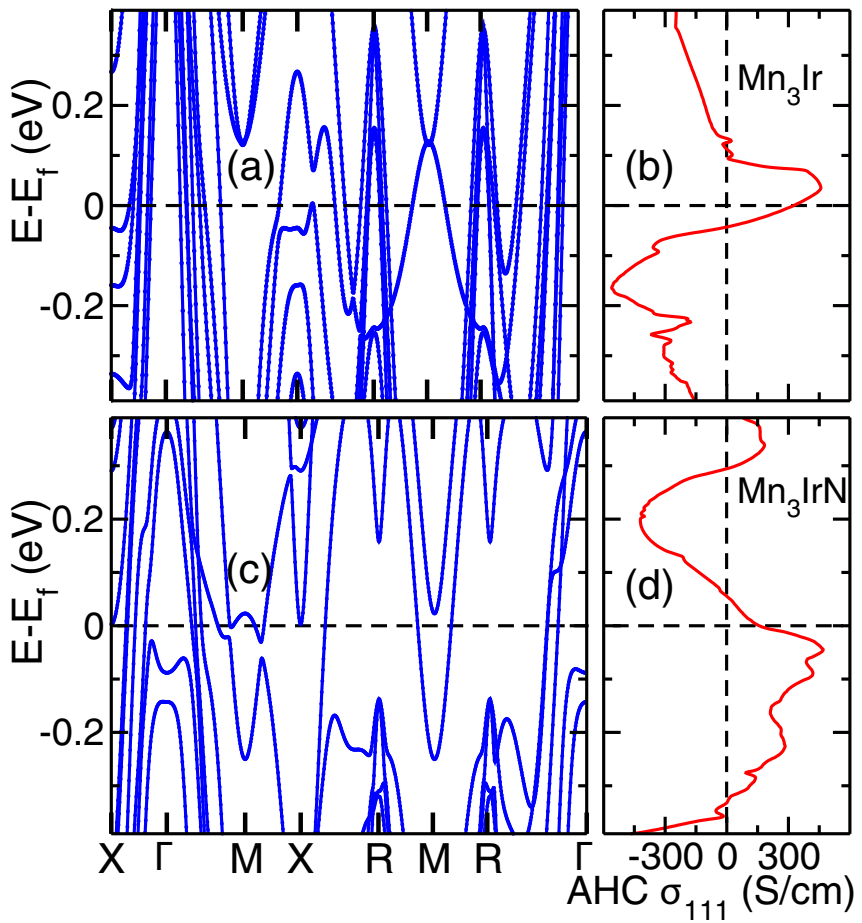

FIG. 9. Calculated band structure and AHC of (a) and (b) $\mathrm{Mn}_{3} \mathrm{Ir}$ and (c) and (d) $\mathrm{Mn}_{3} \mathrm{IrN}$, respectively.

materials for providing exchange bias in hard magnetic films. In the case of $\mathrm{Mn}_{3} \operatorname{Pt}(\mathrm{N}), \Delta E$ changes sign from $-3 \mathrm{meV}$ $\left(\mathrm{Mn}{ }_{3} \mathrm{Pt}\right)$ to $6 \mathrm{meV}\left(\mathrm{Mn}_{3} \mathrm{PtN}\right)$, so that the $\Gamma^{5 g}$ representation becomes the ground state.

According to our calculations, a relatively large epitaxial strain around $5 \%$ is necessary to stabilize a ferrimagnetic ground state in $\mathrm{Mn}_{3} \mathrm{IrN}$ at $0 \mathrm{~K}$ (see Fig. 1 in the Supplemental Material [64]). Surprisingly, the necessary strain in $\mathrm{Mn}_{3} \mathrm{Ir}$ is lower, despite the smaller energy difference between the $\mathrm{NC}$ and ferrimagnetic states in $\mathrm{Mn}_{3} \mathrm{IrN}$, possibly indicating that an intermediate $\mathrm{N}$ concentration is the most suitable for a stabilization of the ferrimagnetic state. Further, we note that for the related compound $\mathrm{Mn}_{3} \mathrm{GaN}$ calculations by Zemen et al. [79] have shown that a combination of strain and temperature leads to a stabilization of a ferrimagnetic ground state. A similar behavior was recently also experimentally observed for a $\mathrm{Mn}_{3} \mathrm{NiN}$ film on a $\mathrm{SrTiO}_{3}$ substrate [80]. In passing we note also that epitaxial strain in $\mathrm{Mn}_{3} X \mathrm{~N}$ as well as in $\mathrm{Mn}_{3} X(X=\mathrm{Rh}, \mathrm{Ir}, \mathrm{Pt})$ is accompanied by a significant net magnetization in the NC ground state, albeit smaller than the one predicted for $\mathrm{Mn}_{3} \mathrm{SnN}$ [81]. This suggests that a piezomagnetic effect may be observed in a wide range of $\mathrm{NC}$ antiferromagnets. A more detailed analysis is beyond the scope of the present high-throughput study and left for future experimental and theoretical work.

The AHC and ANC depend on the spin configuration of the system. The origin of nonvanishing $\mathrm{AHC}$ in $\Gamma^{4 g}$ magnetic ordering has been studied based on symmetry analysis $[82,83]$. Figure 9 shows the calculated band structure and AHC of $\mathrm{Mn}_{3} \mathrm{Ir}$ and $\mathrm{Mn}_{3} \mathrm{IrN}$ in the $\Gamma^{4 g}$ ground state. For both compounds. the calculated AHC $\sigma_{111}$ is significant, with
$315 \mathrm{~S} / \mathrm{cm}$ for $\mathrm{Mn}_{3} \mathrm{Ir}$ and $157 \mathrm{~S} / \mathrm{cm}$ for $\mathrm{Mn}_{3} \mathrm{IrN}$. This is in overall good agreement with a study by Chen et al. in which a moderately smaller AHC was calculated for $\mathrm{Mn}_{3} \mathrm{Ir}$ $(230 \mathrm{~S} / \mathrm{cm})$ [46] but differs in sign and magnitude from a recent AHC calculation by Huyen et al. for $\mathrm{Mn}_{3} \operatorname{IrN}(-573.3$ $\mathrm{S} / \mathrm{cm}$ ) [84]. The AHC has been shown to be highly sensitive to the choice of the magnetization direction [83], which may be a reason for this discrepancy. Based on our calculations, we predict a huge anomalous Nernst effect for $\mathrm{Mn}_{3} \mathrm{Ir}$ and $\mathrm{Mn}_{3} \mathrm{IrN}$. The calculated ANC $\alpha_{111}$ is 6195 and $-4892 \mathrm{~S} /(\mathrm{cm} \mathrm{eV})$ for $\mathrm{Mn}_{3} \mathrm{Ir}$ and $\mathrm{Mn}_{3} \mathrm{IrN}$, respectively, within the range of the best reported antiferromagnetic materials up to now [85-87]. Noteworthily, there is a change in sign of ANC between $\mathrm{Mn}_{3} \mathrm{Ir}$ and $\mathrm{Mn}_{3} \mathrm{IrN}$. Both end members of the $\mathrm{Mn}_{3} \operatorname{IrN}_{x}(0 \leqslant x \leqslant$ 1) series show interesting transport properties. According to our thermodynamic calculations alloys with intermediate $\mathrm{N}$ concentrations are stable, which may be a route for further tuning of the transport properties.

\section{SUMMARY}

In summary we have shown that the interstitial elements $\mathrm{N}, \mathrm{C}$, and $\mathrm{B}$ can have a strong impact on the magnetic properties of cubic compounds and that interstitial alloying can be a fruitful approach to enhance the magnetic properties. For reliable predictions of materials with enhanced properties, phase stability (i.e., calculation of the convex hull including all possible decomposition reactions) is essential. The high-throughput scheme introduced in this paper is a unique computational tool to identify promising magnetic materials in order to guide the design of new magnetic materials.

Based on our calculations, we expect ferromagnetic $\mathrm{Ni}_{3} \mathrm{MnN}_{x}$ (with about 11 at.\% $\mathrm{N}$ ) and the ferrimagnetic compounds $\mathrm{Mn}_{3} \mathrm{SbN}$ and $\mathrm{Mn}_{3} \mathrm{RhN}_{x}$ (with about 30 at.\% $\mathrm{N}$ ) to show bulk magnetic properties with sizable magnetization in the range of $0.5 \mu_{B}-1 \mu_{B} /$ atom and sizable uniaxial MAE in the range of $100-200 \mu \mathrm{eV} /$ atom.

The $\operatorname{Mn}_{3} X(\mathrm{~N})(X=\mathrm{Rh}$, Ir, Pt, and $\mathrm{Sb})$ compounds are identified as materials with strong MAE and a sizable magnetization in a collinear ferrimagnet state. The calculations also show that, with the exception of $\mathrm{Mn}_{3} \mathrm{SbN}$, these materials cannot readily be used as bulk magnets, as their ground state is a noncollinear fully compensated antiferromagnet. As a strong magnetic anisotropy is also present in the noncollinear ground state of these alloys, these materials are, however, of strong interest for multilayer structures where the hard magnetic antiferromagnetic layer is exchange coupled to a soft magnetic layer with large magnetization. Especially for $\mathrm{Mn}_{3} \mathrm{Ir}$, which is already used as an exchange bias material in hard magnetic films, interstitial alloying with $\mathrm{N}$ can be a promising approach to enhance the magnetic properties of a state-of-the-art material. Further, an application of the strong MAE in the ferrimagnetic state may be possible in thin films but requires a more detailed theoretical and experimental investigations. For $\mathrm{Mn}_{3} \operatorname{Ir}$ and $\mathrm{Mn}_{3} \mathrm{IrN}$ we find a significant anomalous Hall effect and a huge anomalous Nernst effect. Variation of the $\mathrm{N}$ concentration may be a route for further 
optimization of the topological transport properties, as the $\mathrm{Mn}_{3} \mathrm{IrN}_{x}$ alloys share the same triangular spin arrangement. Moreover, we have shown that interstitials can also have an impact on the ground-state magnetic structure and can thus be used to tune magnetic transitions, for instance, in magnetocaloric applications. In $\mathrm{Mn}_{3} \mathrm{Sb}(\mathrm{N})$ the magnetic ground state changes from $\mathrm{NC}$ to ferrimagnetic upon alloying with $\mathrm{N}$. For $\mathrm{Mn}_{3} \mathrm{Sb}$, NC, ferrimagnetic, and ferromagnetic magnetic states are very close in energy, indicating that moderate fields may be sufficient to switch from a low to a high magnetization state. This makes $\mathrm{Mn}_{3}$ Sb-based alloys potentially interesting for magnetocaloric applications but requires further studies.

For Mn alloys, our calculations show that the assumption of a ferromagnetic spin alignment, which is currently used in high-throughput databases, is unreliable to predict formation energies and phase stability. The symmetrybased methods applied in this paper to determine the magnetic ground state can pave the way to predictions of the phase stability based on DFT high-throughput calculations for these important alloy systems, which are of interest, e.g., for permanent magnet, magnetocaloric, and spintronics applications.

\section{ACKNOWLEDGMENTS}

We acknowledge fruitful discussions with K. G. Sandeman and Z. Gercsi. Financial support from the Deutsche Forschungsgemeinschaft (DFG, German Research Foundation) through CRC/TRR 270, Project ID 405553726; the German federal state of Hessen through its excellence program LOEWE "RESPONSE"; and the European Community (NOVAMAG) is gratefully acknowledged. The work of J.Z. was supported by the Ministry of Education, Youth and Sports of the Czech Republic from the OP RDE program under the project International Mobility of Researchers MSCA-IF at CTU No. CZ.02.2.69/0.0/0.0/18_070/0010457 and from the Large Infrastructures for Research, Experimental Development and Innovations project IT4Innovations National Supercomputing Center-LM2015070. Calculations for this research were conducted on the Lichtenberg high-performance computer of the TU Darmstadt.
[1] D. Sander, S. O. Valenzuela, D. Makarov, C. H. Marrows, E. E. Fullerton, P. Fischer, J. McCord, P. Vavassori, S. Mangin, P. Pirro, B. Hillebrands, A. D. Kent, T. Jungwirth, O. Gutfleisch, C. G. Kim, and A. Berger, The 2017 magnetism roadmap, J. Phys. D 50, 363001 (2017).

[2] J. Železný, H. Gao, K. Výborný, J. Zemen, J. Mašek, A. Manchon, J. Wunderlich, J. Sinova, and T. Jungwirth, Relativistic Néel-Order Fields Induced by Electrical Current in Antiferromagnets, Phys. Rev. Lett. 113, 157201 (2014).

[3] V. Baltz, A. Manchon, M. Tsoi, T. Moriyama, T. Ono, and Y. Tserkovnyak, Antiferromagnetic spintronics, Rev. Mod. Phys. 90, 015005 (2018).

[4] O. Gutfleisch, M. A. Willard, E. Brück, C. H. Chen, S. G. Sankar, and J. P. Liu, Magnetic materials and devices for the 21st century: stronger, lighter, and more energy efficient, Adv. Mater. 23, 821 (2011).

[5] M. D. Kuz'min, K. P. Skokov, H. Jian, I. Radulov, and O. Gutfleisch, Towards high-performance permanent magnets without rare earths, J. Phys.: Condens. Matter 26, 064205 (2014)

[6] K. Skokov and O. Gutfleisch, Heavy rare earth free, free rare earth and rare earth free magnets-Vision and reality, Scr. Mater. 154, 289 (2018).

[7] S. Curtarolo, G. L. W. Hart, M. B. Nardelli, N. Mingo, S. Sanvito, and O. Levy, The high-throughput highway to computational materials design, Nat. Mater. 12, 191 (2013).

[8] A. Jain, S. P. Ong, G. Hautier, W. Chen, W. D. Richards, S. Dacek, S. Cholia, D. Gunter, D. Skinner, G. Ceder, and K. A. Persson, Commentary: The Materials Project: A materials genome approach to accelerating materials innovation, APL Mater. 1, 011002 (2013).

[9] G. Hautier, A. Jain, S. P. Ong, B. Kang, C. Moore, R. Doe, and G. Ceder, Phosphates as lithium-ion battery cathodes: An evaluation based on high-throughput $a b$ initio calculations, Chem. Mater. 23, 3495 (2011).

[10] J. E. Saal, S. Kirklin, M. Aykol, B. Meredig, and C. Wolverton, Materials design and discovery with high-throughput density functional theory: the open quantum materials database (oqmd), JOM 65, 1501 (2013).

[11] G. K. H. Madsen, Automated search for new thermoelectric materials: The case of LiZnSb, J. Am. Chem. Soc. 128, 12140 (2006).

[12] S. Wang, Z. Wang, W. Setyawan, N. Mingo, and S. Curtarolo, Assessing the Thermoelectric Properties of Sintered Compounds via High-Throughput Ab-Initio Calculations, Phy. Rev X 1, 021012 (2011).

[13] C. Bera, S. Jacob, I. Opahle, N. S. H. Gunda, R. Chmielowski, G. Dennler, and G. K. H. Madsen, Integrated computational materials discovery of silver doped tin sulfide as a thermoelectric material, Phys. Chem. Chem. Phys. 16, 19894 (2014).

[14] L. Yu and A. Zunger, Identification of Potential Photovoltaic Absorbers Based on First-Principles Spectroscopic Screening of Materials, Phys. Rev. Lett. 108, 068701 (2012).

[15] J. D. Bocarsly, E. E. Levin, C. A. C. Garcia, K. Schwennicke, S. D. Wilson, and R. Seshadri, A simple computational proxy for screening magnetocaloric compounds, Chem. Mater. 29, 1613 (2017).

[16] N. Drebov, A. Martinez-Limia, L. Kunz, A. Gola, T. Shigematsu, T. Eckl, P. Gumbsch, and C. Elsässer, Ab initio screening methodology applied to the search for new permanent magnetic materials, New J. Phys. 15, 125023 (2013).

[17] W. Körner, G. Krugel, and C. Elsässer, Theoretical screening of intermetallic $\mathrm{ThMn}_{12}$-type phases for new hard-magnetic compounds with low rare earth content, Sci. Rep. 6, 24686 (2016).

[18] W. Körner, G. Krugel, D. F. Urban, and C. Elsässer, Screening of rare-earth-lean intermetallic 1-11 and 1-11-X compounds of $\mathrm{YNi}_{9} \mathrm{In}_{2}$-type for hard-magnetic applications, Scr. Mater. 154, 295 (2018)

[19] A. Edström, J. Chico, A. Jakobsson, A. Bergman, and J. Rusz, Electronic structure and magnetic properties of $L 1_{0}$ binary alloys, Phys. Rev. B 90, 014402 (2014).

[20] H. C. Herper, Ni-based Heusler compounds: How to tune the magnetocrystalline anisotropy, Phys. Rev. B 98, 014411 (2018). 
[21] O. Y. Vekilova, B. Fayyazi, K. P. Skokov, O. Gutfleisch, C. Echevarria-Bonet, J. M. Barandiarán, A. Kovacs, J. Fischbacher, T. Schrefl, O. Eriksson, and H. C. Herper, Tuning the magnetocrystalline anisotropy of $\mathrm{Fe}_{3} \mathrm{Sn}$ by alloying, Phys. Rev. B 99, 024421 (2019).

[22] B. Fayyazi, K. P. Skokov, T. Faske, I. Opahle, M. Duerrschnabel, T. Helbig, I. Soldatov, U. Rohrmann, L. MolinaLuna, K. Güth, H. Zhang, W. Donner, R. Schäfer, and O. Gutfleisch, Experimental and computational analysis of binary Fe-Sn ferromagnetic compounds, Acta Mater. 180, 126 (2019).

[23] S. Sanvito, C. Oses, J. Xue, A. Tiwari, M. Zic, T. Archer, P. Tozman, M. Venkatesan, M. Coey, and S. Curtarolo, Accelerated discovery of new magnets in the Heusler alloy family, Sci. Adv. 3, e1602241 (2017).

[24] J. Balluff, K. Diekmann, G. Reiss, and M. Meinert, Highthroughput screening for antiferromagnetic Heusler compounds using density functional theory, Phys. Rev. Mater. 1, 034404 (2017).

[25] S. Arapan, P. Nieves, and S. Cuesta-López, A high-throughput exploration of magnetic materials by using structure predicting methods, J. Appl. Phys. 123, 083904 (2018).

[26] H. K. Singh, Z. Zhang, I. Opahle, D. Ohmer, Y. Yao, and H. Zhang, high-throughput screening of magnetic antiperovskites, Chem. Mater. 30, 6983 (2018).

[27] J. Kübler, K. H. Höck, J. Sticht, and A. R. Williams, Density functional theory of non-collinear magnetism, J. Phys. F 18, 469 (1988).

[28] W. Zhang, P. Kharel, S. Valloppilly, L. Yue, and D. J. Sellmyer, High-energy-product MnBi films with controllable anisotropy, Phys. Status Solidi B 252, 1934 (2015).

[29] J. Liu, T. Gottschall, K. P. Skokov, J. D. Moore, and O. Gutfleisch, Giant magnetocaloric effect driven by structural transitions, Nat. Mater. 11, 620 (2012).

[30] D. Ohmer, I. Opahle, H. K. Singh, and H. Zhang, Stability predictions of magnetic $\mathrm{M}_{2} \mathrm{AX}$ compounds, J. Phys. Condens. Matter 31, 405902 (2019).

[31] Q. Gao, I. Opahle, and H. Zhang, High-throughput screening for spin-gapless semiconductors in quaternary Heusler compounds, Phys. Rev. Mater. 3, 024410 (2019).

[32] A. Payne, G. Avendaño-Franco, E. Bousquet, and A. H. Romero, Firefly algorithm applied to noncollinear magnetic phase materials prediction, J. Chem. Theory Comput. 14, 4455 (2018).

[33] M. K. Horton, J. H. Montoya, M. Liu, and K. A. Persson, highthroughput prediction of the ground-state collinear magnetic order of inorganic materials using density functional theory, $\mathrm{Npj}$ Comput. Mater. 5, 64 (2019).

[34] J. Coey and H. Sun, Improved magnetic properties by treatment of iron-based rare earth intermetallic compounds in ammonia, J. Magn. Magn. Mater. 87, L251 (1990).

[35] L. Steinbeck, M. Richter, U. Nitzsche, and H. Eschrig, Ab initio calculation of electronic structure, crystal field, and intrinsic magnetic properties of $\mathrm{Sm}_{2} \mathrm{Fe}_{17}, \mathrm{Sm}_{2} \mathrm{Fe}_{17} \mathrm{~N}_{3}, \mathrm{Sm}_{2} \mathrm{Fe}_{17} \mathrm{C}_{3}$, and $\mathrm{Sm}_{2} \mathrm{Co}_{17}$, Phys. Rev. B 53, 7111 (1996).

[36] H. Zhang, I. Dirba, T. Helbig, L. Alff, and O. Gutfleisch, Engineering perpendicular magnetic anisotropy in Fe via interstitial nitrogenation: N choose K, APL Mater. 4, 116104 (2016).

[37] M. Gajdzik, C. Sürgers, M. Kelemen, and H. v. Löhneysen, Ferromagnetism in carbon-doped $\mathrm{Mn}_{5} \mathrm{Si}_{3}$ films, J. Appl. Phys. 87, 6013 (2000).
[38] J. Lyubina, O. Gutfleisch, M. D. Kuz'min, and M. Richter, $\mathrm{La}(\mathrm{Fe}, \mathrm{Si})_{13}$-based magnetic refrigerants obtained by novel processing routes, J. Magn. Magn. Mater. 320, 2252 (2008).

[39] N. T. Trung, L. Zhang, L. Caron, K. H. J. Buschow, and E. Brück, Giant magnetocaloric effects by tailoring the phase transitions, Appl. Phys. Lett. 96, 172504 (2010).

[40] J. Lyubina, I. Opahle, K.-H. Müller, O. Gutfleisch, M. Richter, M. Wolf, and L. Schultz, Magnetocrystalline anisotropy in $\mathrm{L} 1_{0}$ FePt and exchange coupling in $\mathrm{FePt} / \mathrm{Fe}_{3} \mathrm{Pt}$ nanocomposites, J. Phys.: Condens. Matter 17, 4157 (2005).

[41] N. Hai, N. Dempsey, and D. Givord, Hard magnetic Fe-Pt alloys prepared by cold-deformation, in Occasion of the Hanoi International Symposium on Magnetism: Cryolab 25, special issue of J. Magn. Magn. Mater. 262, 353 (2003).

[42] L. Szunyogh, B. Lazarovits, L. Udvardi, J. Jackson, and U. Nowak, Giant magnetic anisotropy of the bulk antiferromagnets IrMn and $\mathrm{IrMn}_{3}$ from first principles, Phys. Rev. B 79, 020403(R) (2009).

[43] K.-I. Imakita, M. Tsunoda, and M. Takahashi, Giant exchange anisotropy observed in $\mathrm{Mn}$-Ir/Co-Fe bilayers containing ordered $\mathrm{Mn}_{3}$ Ir phase, Appl. Phys. Lett. 85, 3812 (2004).

[44] A. Kohn, A. Kovács, R. Fan, G. J. McIntyre, R. C. C. Ward, and J. P. Goff, The antiferromagnetic structures of $\mathrm{IrMn}_{3}$ and their influence on exchange-bias, Sci. Rep. 3, 2412 (2013).

[45] T. Shimizu, T. Shibayama, K. Asano, and K. Takenaka, Giant magnetostriction in tetragonally distorted antiperovskite manganese nitrides, J. Appl. Phys. 111, 07A903 (2012).

[46] H. Chen, Q. Niu, and A. H. MacDonald, Anomalous Hall Effect Arising from Noncollinear Antiferromagnetism, Phys. Rev. Lett. 112, 017205 (2014).

[47] Z. Q. Liu, H. Chen, J. M. Wang, J. H. Liu, K. Wang, Z. X. Feng, H. Yan, X. R. Wang, C. B. Jiang, J. M. D. Coey, and A. H. MacDonald, Electrical switching of the topological anomalous Hall effect in a non-collinear antiferromagnet above room temperature, Nat. Electron. 1, 172 (2018).

[48] W. Zhang, W. Han, S.-H. Yang, Y. Sun, Y. Zhang, B. Yan, and S. S. P. Parkin, Giant facet-dependent spin-orbit torque and spin Hall conductivity in the triangular antiferromagnet $\mathrm{IrMn}_{3}$, Sci. Adv. 2, e1600759 (2016).

[49] S. V. Gallego, J. M. Perez-Mato, L. Elcoro, E. S. Tasci, R. M. Hanson, K. Momma, M. I. Aroyo, and G. Madariaga, MAGNDATA: Towards a database of magnetic structures. I. The commensurate case, J. Appl. Crystallogr. 49, 1750 (2016).

[50] I. Opahle, G. K. H. Madsen, and R. Drautz, High throughput density functional investigations of the stability, electronic structure and thermoelectric properties of binary silicides, Phys. Chem. Chem. Phys. 14, 16197 (2012).

[51] I. Opahle, A. Parma, E. J. McEniry, R. Drautz, and G. K. H. Madsen, High-throughput study of the structural stability and thermoelectric properties of transition metal silicides, New J. Phys. 15, 105010 (2013).

[52] G. Kresse and J. Furthmüller, Efficient iterative schemes for ab initio total-energy calculations using a plane-wave basis set, Phys. Rev. B 54, 11169 (1996).

[53] G. Kresse and D. Joubert, From ultrasoft pseudopotentials to the projector augmented-wave method, Phys. Rev. B 59, 1758 (1999).

[54] K. Koepernik and H. Eschrig, Full-potential nonorthogonal local-orbital minimum-basis band-structure scheme, Phys. Rev. B 59, 1743 (1999). 
[55] Q. Wu, S. Zhang, H.-F. Song, M. Troyer, and A. A. Soluyanov, WannierTools: An open-source software package for novel topological materials, Comput. Phys. Commun. 224, 405 (2018).

[56] A. A. Mostofi, J. R. Yates, Y.-S. Lee, I. Souza, D. Vanderbilt, and N. Marzari, wannier90: A tool for obtaining maximallylocalised Wannier functions, Comput. Phys. Commun. 178, 685 (2008).

[57] D. Xiao, M.-C. Chang, and Q. Niu, Berry phase effects on electronic properties, Rev. Mod. Phys. 82, 1959 (2010).

[58] J. P. Perdew, K. Burke, and M. Ernzerhof, Generalized Gradient Approximation Made Simple, Phys. Rev. Lett. 77, 3865 (1996).

[59] J. P. Perdew and Y. Wang, Accurate and simple analytic representation of the electron-gas correlation energy, Phys. Rev. B 45, 13244 (1992).

[60] S. R. Bahn and K. W. Jacobsen, An object-oriented scripting interface to a legacy electronic structure code, Comput. Sci. Eng. 4, 56 (2002).

[61] A. H. Larsen, J. J. Mortensen, J. Blomqvist, I. E. Castelli, R. Christensen, M. Dułak, J. Friis, M. N. Groves, B. Hammer, C. Hargus, E. D. Hermes, P. C. Jennings, P. B. Jensen, J. Kermode, J. R. Kitchin, E. L. Kolsbjerg, J. Kubal, K. Kaasbjerg, S. Lysgaard, J. B. Maronsson et al., The atomic simulation environment-A Python library for working with atoms, J. Phys.: Condens. Matter 29, 273002 (2017).

[62] Materials Project, https://materialsproject.org.

[63] T. Yamashita, H. Takizawa, T. Sasaki, K. Uheda, and T. Endo, $\mathrm{Mn}_{3} \mathrm{Sb}$ : A new $\mathrm{L1}_{2}$-type intermetallic compound synthesized under high-pressure, J. Alloys Compd. 348, 220 (2003).

[64] See Supplemental Material at http://link.aps.org/supplemental/ 10.1103/PhysRevResearch.2.023134 for details of the calculated properties of alloys close to the convex hull and the effect of epitaxial strain on $\mathrm{Mn}_{3} X(\mathrm{~N})(X=\mathrm{Rh}, \mathrm{Ir}, \mathrm{Pt})$ alloys.

[65] M. Ellner, S. Kek, and B. Predel, Zur existenz einer Phase $\mathrm{Co}_{3} \mathrm{Al}$ vom $\mathrm{Cu}_{3} \mathrm{Au}-\mathrm{Strukturtyp}$, J. Alloys Compd. 189, 245 (1992).

[66] E. Krén, G. Kádár, L. Pál, J. Sólyom, P. Szabó, and T. Tarnóczi, Magnetic structures and exchange interactions in the mn-pt system, Phys. Rev. 171, 574 (1968).

[67] I. Tomeno, H. N. Fuke, H. Iwasaki, M. Sahashi, and Y. Tsunoda, Magnetic neutron scattering study of ordered $\mathrm{Mn}_{3} \mathrm{Ir}$, J. Appl. Phys. 86, 3853 (1999).

[68] V. M. Ryzhkovskii, V. S. Goncharov, S. S. Agafonov, V. P. Glazkov, V. A. Somenkov, A. P. Sazonov, and A. T. Senishin, Magnetic ordering in $\mathrm{Mn}_{3}$ Sb determined by neutron diffraction data, J. Surf. Invest.: X-Ray, Synchrotron Neutron Tech. 5, 109 (2011).

[69] M. Budzynski, V. S. Goncharov, V. I. Mitsiuk, Z. Surowiec, and T. M. Tkachenka, Mössbauer Study of Cubic Phase in the Mn-Sb System, in Proceedings of the 41st Polish Seminar on Positron Annihilation, Lublin, September 9-13, 2013, special issue of Acta Phys. Pol. A 125, 850 (2014).

[70] Open Quantum Materials Database, http://oqmd.org/.

[71] K. Selte, A. Kjekshus, A. F. Andresen, and W. B. Pearson, Equiatomic transition metal alloys of manganese. VII. A neutron diffraction study of magnetic ordering in the IrMn phase, Acta Chem. Scand. 22, 3039 (1968).
[72] D. Fruchart and E. F. Bertaut, Magnetic studies of the metallic perovskite-type compounds of manganese, J. Phys. Soc. Jpn. 44, 781 (1978).

[73] W. Cao, B. He, C. Liao, L. Yang, L. Zeng, and C. Dong, Preparation and properties of antiperovskite-type nitrides: $\mathrm{InNNi}_{3}$ and $\mathrm{InNCO}_{3}$, J. Solid State Chem. 182, 3353 (2009).

[74] G. W. Wiener and J. A. Berger, Structure and magnetic properties of some transition metal nitrides, JOM 7, 360 (1955).

[75] L. Huetter and H. Stadelmaier, Ternary carbides of transition metals with aluminum and magnesium, Acta Metall. 6, 367 (1958).

[76] A. Houben, J. Burghaus, and R. Dronskowski, The ternary nitrides $\mathrm{GaFe}_{3} \mathrm{~N}$ and $\mathrm{AlFe}_{3} \mathrm{~N}$ : Improved synthesis and magnetic properties, Chem. Mater. 21, 4332 (2009).

[77] T. Scholz and R. Dronskowski, Improved ammonolytic synthesis, structure determination, electronic structure, and magnetic properties of the solid solution $\mathrm{Sn}_{x} \mathrm{Fe}_{4-x} \mathrm{~N}(0 \leqslant x \leqslant 0.9)$, Inorg. Chem. 54, 8800 (2015).

[78] S. Ener, M. Fries, F. Hammerath, I. Opahle, E. Simon, P. Fritsch, S. Wurmehl, H. Zhang, and O. Gutfleisch, Magnetic and magnetocaloric properties of the $\mathrm{Co}_{2-x} \mathrm{Mn}_{x} \mathrm{~B}$ system by experiment and density functional theory, Acta Mater. 165, 270 (2019).

[79] J. Zemen, E. Mendive-Tapia, Z. Gercsi, R. Banerjee, J. B. Staunton, and K. G. Sandeman, Frustrated magnetism and caloric effects in Mn-based antiperovskite nitrides: $A b$ initio theory, Phys. Rev. B 95, 184438 (2017).

[80] D. Boldrin, F. Johnson, R. Thompson, A. P. Mihai, B. Zou, J. Zemen, J. Griffiths, P. Gubeljak, K. L. Ormandy, P. Manuel, D. D. Khalyavin, B. Ouladdiaf, N. Qureshi, P. Petrov, W. Branford, and L. F. Cohen, The biaxial strain dependence of magnetic order in spin frustrated $\mathrm{Mn}_{3} \mathrm{NiN}$ thin films, Adv. Funct. Mater. 29, 1902502 (2019).

[81] J. Zemen, Z. Gercsi, and K. G. Sandeman, Piezomagnetism as a counterpart of the magnetovolume effect in magnetically frustrated Mn-based antiperovskite nitrides, Phys. Rev. B 96, 024451 (2017).

[82] G. Gurung, D.-F. Shao, T. R. Paudel, and E. Y. Tsymbal, Anomalous Hall conductivity of noncollinear magnetic antiperovskites, Phys. Rev. Mater. 3, 044409 (2019).

[83] I. Samathrakis and H. Zhang, Piezospintronic effect in antiperovskite $\mathrm{Mn}_{3} \mathrm{GaN}$, arXiv:1905.11798.

[84] V. T. N. Huyen, M.-T. Suzuki, K. Yamauchi, and T. Oguchi, Topology analysis for anomalous Hall effect in the noncollinear antiferromagnetic states of $\mathrm{Mn}_{3} A \mathrm{~N}(A=\mathrm{Ni}, \mathrm{Cu}, \mathrm{Zn}, \mathrm{Ga}, \mathrm{Ge}$, Pd, In, Sn, Ir, Pt), Phys. Rev. B 100, 094426 (2019).

[85] G.-Y. Guo and T.-C. Wang, Large anomalous Nernst and spin Nernst effects in the noncollinear antiferromagnets $\operatorname{Mn}_{3} X(X=$ Sn, Ge, Ga), Phys. Rev. B 96, 224415 (2017).

[86] X. Zhou, J.-P. Hanke, W. Feng, S. Blügel, Y. Mokrousov, and Y. Yao, Giant anomalous Nernst effect in noncollinear antiferromagnetic Mn-based antiperovskite nitrides, Phys. Rev. Mater. 4, 024408 (2020).

[87] D. Boldrin, I. Samathrakis, J. Zemen, A. Mihai, B. Zou, F. Johnson, B. D. Esser, D. W. McComb, P. K. Petrov, H. Zhang, and L. F. Cohen, Anomalous Hall effect in noncollinear antiferromagnetic $\mathrm{Mn}_{3} \mathrm{NiN}$ thin films, Phys. Rev. Mater. 3, 094409 (2019). 